\title{
The Galactic Faraday depth sky revisited ${ }^{\star}$
}

\author{
Sebastian Hutschenreuter ${ }^{1,2}$ and Torsten A. Enßlin ${ }^{1,2}$ \\ ${ }^{1}$ Max Planck Institute for Astrophysics, Karl-Schwarzschildstr. 1, 85741 Garching, Germany \\ e-mail: hutsch@mpa-garching.mpg.de \\ ${ }^{2}$ Ludwig-Maximilians-Universität München, Geschwister-Scholl-Platz 1, 80539 Munich, Germany
}

Received 16 March 2019 / Accepted 1 September 2019

\begin{abstract}
Context. The Galactic Faraday depth sky is a tracer for both the Galactic magnetic field and the thermal electron distribution. It was previously reconstructed from polarimetric measurements of extra-Galactic point sources.

Aims. Here we improve on these works by using an updated inference algorithm and by taking into account the electron emission measure as traced by free-free emission measured by the Planck survey. In the future the data situation will improve drastically thanks to the next generation Faraday rotation measurements from the SKA and its pathfinders. Anticipating this, a further aim of this paper is to update the map reconstruction method with some of the latest developments in Bayesian imaging.

Methods. To this end we made use of information field theory, an inference scheme that is particularly powerful in cases of noisy and incomplete data.

Results. We demonstrate the validity of the new algorithm by applying it to an existing data compilation. Even though we used exactly the same data set, a number of novel findings are made; for example, a non-parametric reconstruction of an overall amplitude field resembles the free-free emission measure map of the Galaxy. Folding this emission measure map into the analysis provides more detailed predictions. The joint inference enables us to identify regions with deviations from the assumed correlations between the emission measure and Faraday data, thereby pointing us to Galactic structures with distinguishably different physics. We find evidence for an alignment of the magnetic field within the lines of sight along both directions of the Orion arm.
\end{abstract}

Key words. ISM: magnetic fields - ISM: structure

\section{Introduction}

The Faraday rotation effect is one of the primary sources of information on astrophysical and cosmological magnetic fields. This includes the fields of planets (Boudjada \& Lecacheux 1991), stars (Kooi et al. 2017), other galaxies (Gießübel et al. 2013), and galaxy clusters (Dreher et al. 1987), as well as more curious objects such as radio jets, lobes, and relics (e.g., Bonafede et al. 2013). In particular, the study of the Faraday rotation induced by the Milky Way magnetic field is of twofold importance. First of all, it is an interesting research topic on its own due its connection to the formation and structure of our home galaxy; furthermore, it constitutes a non-negligible foreground component. All polarized light stemming from cosmological sources passes through the Galaxy and interacts with the Galactic magnetic field, which affects its polarization direction. Accurate and highly resolved Galactic foreground templates are therefore a necessary condition for any precision measurement of extra-Galactic magnetic fields via Faraday rotation.

Multiple efforts have been made to to map the Galactic Faraday sky, quite a few of them already to remarkable accuracy (e.g., in Frick et al. 2001; Johnston-Hollitt et al. 2004; Dineen \& Coles 2005; Xu et al. 2006; Oppermann et al. 2012, 2015, and Xu \& Han 2014). The approach in Oppermann et al. (2012, hereafter NO12) is especially important to us as we rely on the same theoretical framework for our inference algorithms. We refer to its successor paper Oppermann et al. (2015, hereafter NO15) from here on.

* The reconstructed Faraday sky is also available at the CDS via anonymous ftp to cdsarc.u-strasbg.fr $(130.79 .128 .5)$ or via http://cdsarc.u-strasbg.fr/viz-bin/cat/J/A+A/633/A150
Our goal in this work is to further sharpen our knowledge on the Faraday sky by exploiting correlations to the electron emission measure (EM) as traced by bremsstrahlung measured from electron proton interaction in the interstellar medium, commonly known as the Galactic free-free emission. This is well motivated by observation as well as physical considerations, as we outline later on. In a more general context we hope to demonstrate an interesting test case of multi-wavelength astronomy, and show that the synthesis of long existing data sets can still yield undiscovered information, under the condition that our a priori physical knowledge is employed and the data is treated in a consistent way. Our main result, the revised Faraday sky including the free-free data (hereafter reconstruction I) is shown in Fig. 1a, and the revised map without free-free data in Fig. 1b (reconstruction II).

The paper is structured as follows. In Sect. 2 we develop our updated reconstruction method. This involves an amplitude field representing the overall Galactic structure and a sign field representing magnetic reversals and smaller scale structures. It turns out that the reconstructed amplitude field resembles the freefree EM of the Galaxy, which is physically plausible. In order to take advantage of this, we extend the method to fold in the measurements of the free-free EM sky in Sect. 3. We conclude in Sect. 4. Mathematical details of the method can be found in the appendix.

\section{Improving the inference of the Faraday sky}

\subsection{The physics}

The rotation angle $\Delta_{\lambda}$ of the polarization plane of linearly polarized light due to Faraday rotation can be written in most 


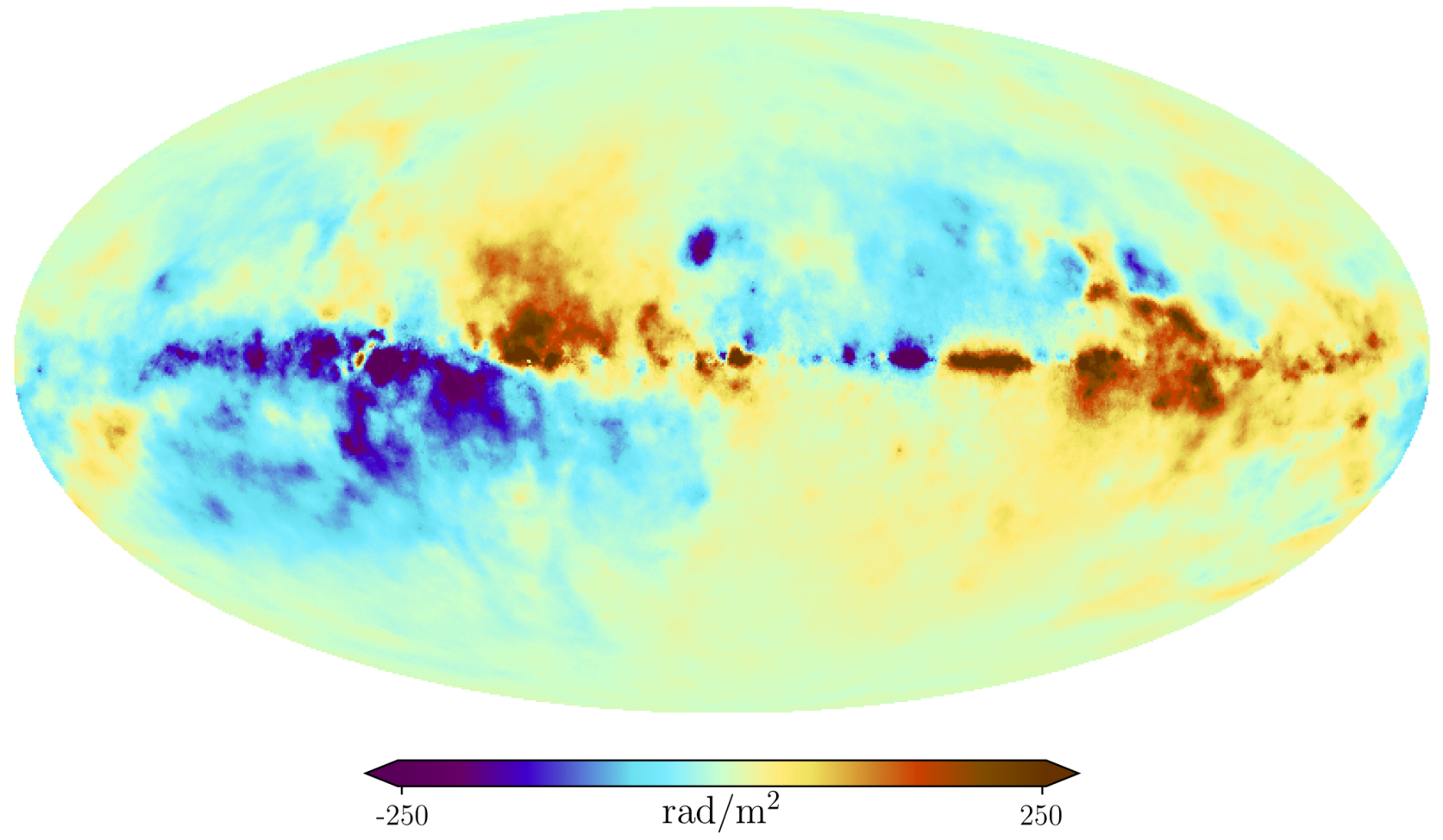

(a)

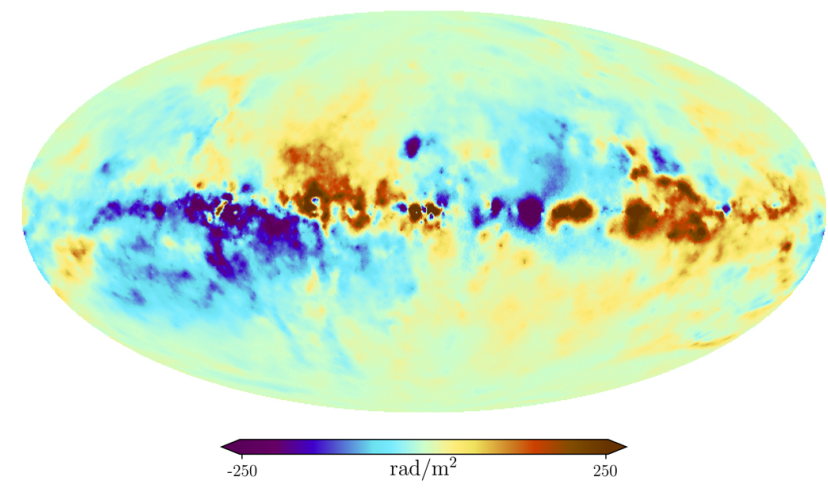

(b)

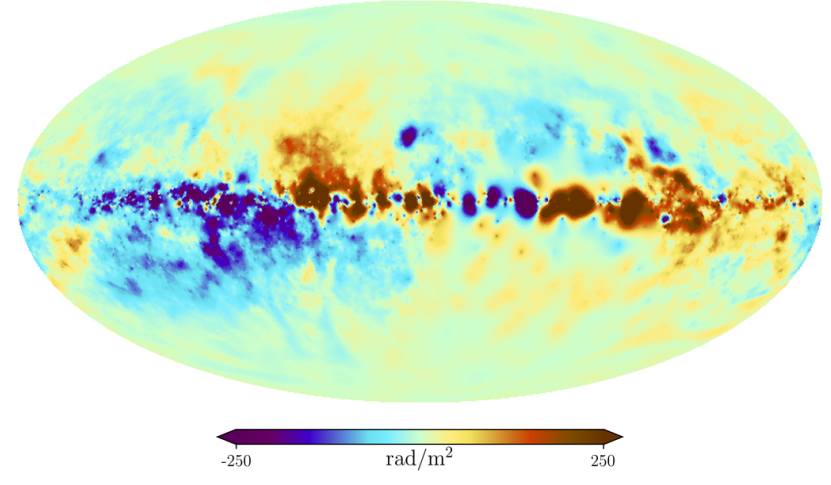

(c)

Fig. 1. Reconstructions of the Faraday sky. Panel a: our main result, the posterior mean of reconstruction I. This map uses the free-free EM map as a proxy for the Faraday amplitude. The model also contains fields that translate the free-free map into a Faraday amplitude, thereby balancing effects that support and those that disturb a direct relation of these two quantities. Panel $b$ : posterior mean of reconstruction II, without additional free-free data. Panel $c$ : posterior mean of the (NO15) reconstruction. The differences in the three maps are shown in Fig. 2. These maps and all maps following in this paper are presented in Galactic coordinates centered at $(l, b)=\left(0^{\circ}, 0^{\circ}\right)$. The results are available online.

astrophysical settings as (Burn 1966)

$\Delta_{\lambda}=\phi\left(n_{\mathrm{th}}, B_{\mathrm{LOS}}\right) \lambda^{2}$

where $\lambda$ is the wavelength at which the light is observed. The Faraday depth $\phi$ depends on the thermal electron density $n_{\text {th }}$ and the line-of-sight (LOS) component of the magnetic field $B_{\mathrm{LOS}}$. More specifically for a polarized extra-Galactic source shining through the interstellar medium, the Galactic part of the Faraday depth $\phi_{\text {gal }}$ can be written as (Brentjens \& de Bruyn 2005)

$\phi_{\text {gal }}=\frac{e^{3}}{2 \pi m_{\mathrm{e}}^{2} c^{4}} \int_{\mathrm{LOS}} \mathrm{d} l n_{\mathrm{th}} B_{\text {gal }, \mathrm{LOS}}$, where $B_{\text {gal }}$ is the Galactic magnetic field, $e$ is the electron charge, $m_{\mathrm{e}}$ is the electron mass, and $c$ the speed of light. Contributions to the observed rotation angle not only come from the Galactic magnetic field, but also contain extra-Galactic, potentially source internal contributions. The Faraday rotation measure (RM) is commonly obtained by fitting the rotation angle defined in Eq. (1) over $\lambda^{2}$ and calculating the slope. Only in cases with negligible foreground emission is this slope equal to $\phi$. Otherwise, the resulting observed curve in $\lambda^{2}$ space will not be linear and $\phi$ must be obtained via a spectral analysis technique commonly known as RM synthesis (Brentjens \& de Bruyn 2005). In this work we use data points from both techniques and therefore 


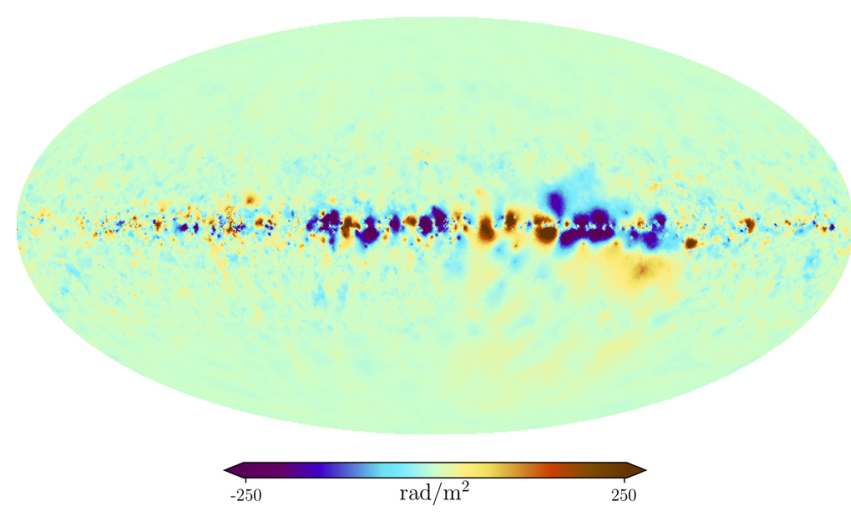

(a)

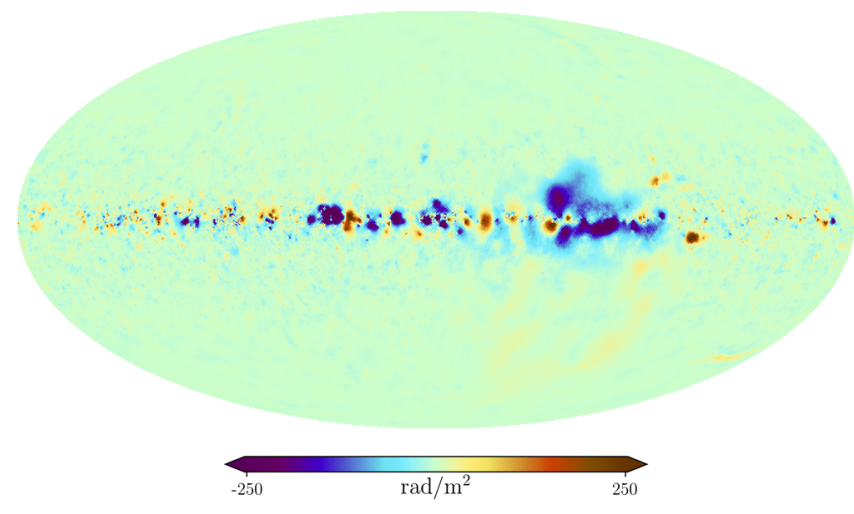

(b)

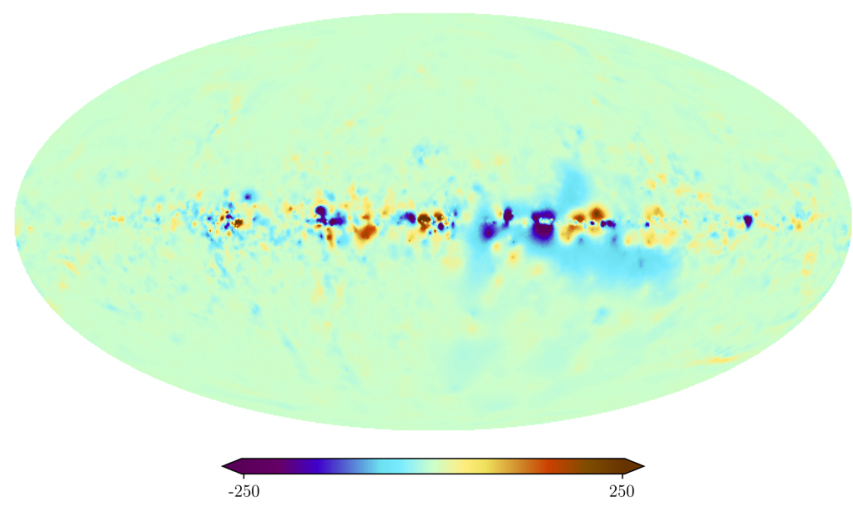

(c)

Fig. 2. Differences in the reconstructions of the Faraday sky shown in Fig. 1. Panel a: difference between the NO15 map and reconstruction I. Panel $b$ : difference between the NO15 map and reconstruction II. Panel $c$ : difference between the Faraday depth skies resulting from reconstruction I and reconstruction II.

use the term "Faraday data" for both RM data and RM synthesis data, in the spirit of NO12. Similar to the approach taken by NO12, it will be helpful to separate the Faraday depth signal into

$\phi=\phi_{\text {gal }}+\phi_{\text {etc }}$.

The term $\phi_{\text {gal }}$ describes the aforementioned Galactic contributions, and $\phi_{\text {etc }}$ describes other contributions, for example extra-Galactic, source intrinsic, or ionospheric. Our goal in this work is the estimation of $\phi_{\text {gal }}$ as a correlated field on the sky. This implies, at the very least, the necessity for a stochastic estimation of the other contributions. The connection of $\phi$ to the observed data $d_{\phi}$ is the topic of the next section.

\subsection{Data}

A measurement of the Galactic Faraday depth can be modeled via

$d_{\phi}=\mathcal{R}_{\phi} \phi+n_{\phi}$,

where we ignore for the moment the potential non-Galactic contributions mentioned in Eq. (3). The quantity $n_{\phi}$ in Eq. (4) is the measurement noise. The operator $\mathcal{R}_{\phi}$ is called the response and describes the noiseless measurement process translating the signal into data space. In this case, the evaluation of the response at the location of source $i$ is sufficiently described via

$[\mathcal{R} \phi]_{i}=\int_{S^{2}} \mathrm{~d} \widehat{e} \phi(\widehat{e}) \delta\left(\widehat{e}-\widehat{e}_{i}\right)$,

with $\widehat{e}$ being the radial unit vector on the two-sphere. The measurement noise can be assumed to be independent and Gaussian distributed according to

$\mathcal{P}\left(n_{\phi}\right)=\mathcal{G}\left(n_{\phi}, N_{\phi}\right)=\frac{1}{\sqrt{\left|2 \pi N_{\phi}\right|}} e^{-\frac{1}{2} n_{\phi} N_{\phi}^{-1} n_{\phi}^{\dagger}}$,

with zero mean and known noise covariance $N_{\phi}$. The noise will play an important role for our estimation of non-Galactic contributions to $\phi$, which is discussed in more detail in Sect. 2.3.2. For the moment we assume $N_{\phi}$ to be simply given by the observational measurement uncertainties, therefore a diagonal matrix in data space.

As we are conducting a Bayesian inference, our main task is the evaluation of the posterior probability distribution. An integral part of this distribution is the likelihood, which in our case is best described by the following Gaussian:

$\mathcal{P}\left(d_{\phi} \mid \phi\right)=\mathcal{G}\left(d_{\phi}-\mathcal{R}_{\phi} \phi, N_{\phi}\right)$.

The likelihood will be further determined by the specific modeling of $\phi$, which we will present in Sect. 4 together with the different prior terms.

The data set used in this work is exactly the same compilation of Faraday rotation measures as used in NO12 and is mostly publicly accessible ${ }^{1}$. This compilation contains rotation measures, corresponding standard deviations, and source positions for 41632 sources $^{2}$. As was done in NO15, we multiplied

\footnotetext{
1 https://wwwmpa.mpa-garching.mpg.de/ ensslin/ research/data/faraday_revisited.html

2 Bonafede et al. (2010); Broten et al. (1988); Brown et al. (2003, 2007); Taylor et al. (2003); Haverkorn et al. (2006); McClure-Griffiths et al. (2005); Clarke et al. (2001); Clarke (2004); Clegg et al. (1992); Feain et al. (2009, 2011); Gaensler et al. (2001); Hammond (2011, priv. comm.); Heald et al. (2009); Braun et al. (2007); Hennessy et al. (1989); Johnston-Hollitt (2011, priv. comm.); Johnston-Hollitt (2003); Johnston-Hollitt \& Ekers (2004); Kato et al. (1987); Kim et al. (1991); Klein et al. (2003); Gregorini et al. (1998); Vigotti et al. (1999); Lawler \& Dennison (1982); Dennison (1979); Mao et al. (2008, 2010, 2012); Gaensler et al. (2005); Minter \& Spangler (1996); Oren \& Wolfe (1995); O’Sullivan (2011, priv. comm.); Roy et al. (2005); Rudnick \& Jones (1983); Schnitzeler (2011, priv. comm.); Simard-Normandin et al. (1981); Tabara \& Inoue (1980); Taylor et al. (2009); Condon et al. (1998); Van Eck et al. (2011); Wrobel (1993).
} 


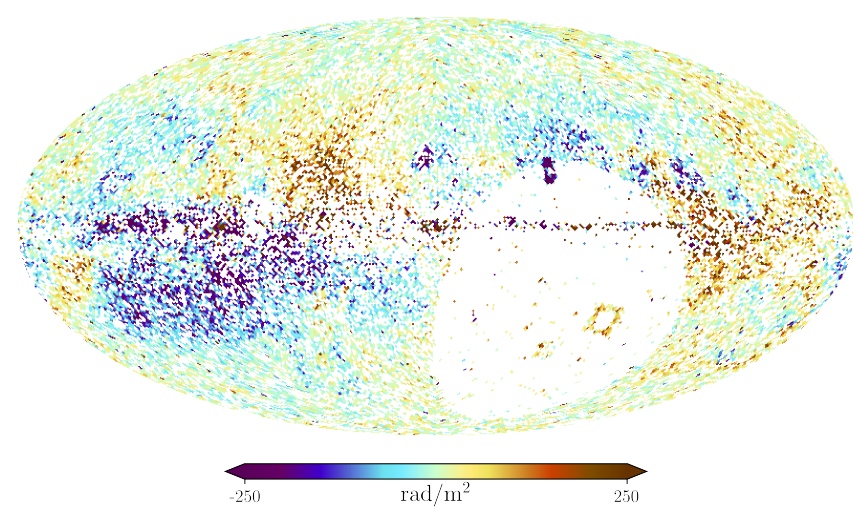

(a)

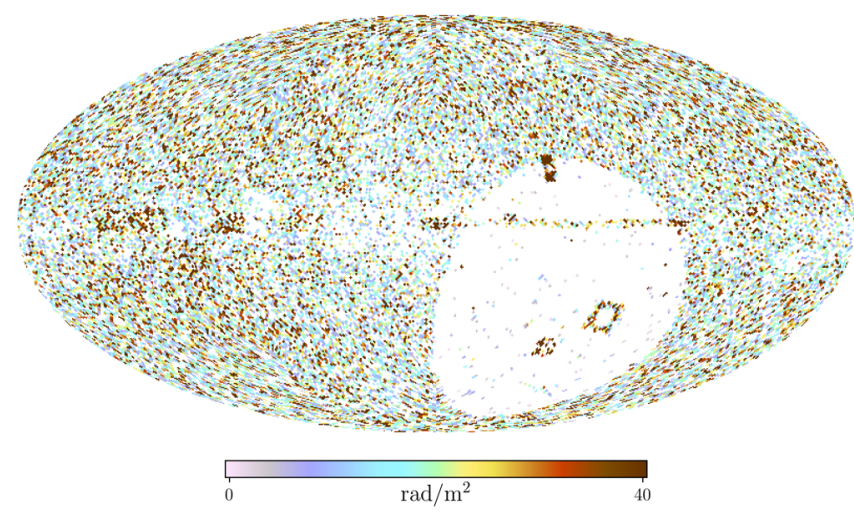

(b)

Fig. 3. Sky map of the Faraday data set and its errors. Panel a: Faraday catalog used in this work projected on the sky. Panel $b$ : the corresponding uncertainties. The region corresponding to the terrestrial south pole is only weakly constrained by data, except for the Galactic disk.

the uncertainties of the Taylor et al. (2009) (sub-)data set with 1.22 according to Stil et al. (2011). We note that Vernstrom et al. (2019) argue against this generic increase of the uncertainties, and in contrast favor a decrease at least for a subset of the Taylor catalog. Furthermore it was found by Ma et al. (2019) that some of the data points of the same catalog suffer from $n \pi$ ambiguities, which means that the reported RMs can be offset by multiples of $\pm 652.9 \mathrm{rad} \mathrm{m}^{-2}$. We discuss in the model section how we deal with such systematic effects in the data. No additional polarimetric information, such as Faraday spectra, was used in this work. The data and the corresponding standard deviations are shown in Figs. 3a and b. Next, we specify the modeling of $\phi$ in Eq. (4). We will also explain the treatment of the non-Galactic components of $d_{\phi}$.

\subsection{Models}

\subsubsection{The sky model}

The results of the previous reconstruction (NO15) indicate that the Faraday sky has two characteristics that necessarily require modeling for an optimal inference. First, the sign of the sky field changes rapidly, which reflects the rather abrupt directional changes of the LOS magnetic field component. Second, the absolute value of the signal varies over two orders of magnitude, most notably the Galactic disk has a much stronger signal than the Galactic poles. For these reasons, we chose to parametrize $\phi: S^{2} \rightarrow \mathbb{R}$ as a point-wise product between two fields living on the sky, or explicitly

$\phi \equiv \chi e^{\rho} \frac{\mathrm{rad}}{\mathrm{m}^{2}}$,

where $\chi: S^{2} \rightarrow \mathbb{R}$ is called the sign field, as it is able to capture the information on the sign of the Faraday depth, and $\rho: S^{2} \rightarrow \mathbb{R}$ is called the amplitude field, as $e^{\rho}$ is strictly positive after the exponentiation and is able to model the large amplitude variations of the Faraday sky over orders of magnitude. Both fields are unitless. This is a more generic approach than in NO12 where, instead of an amplitude field, a profile function was used to capture the latitudinal dependence of the overall Galactic Faraday dispersion profile. We note that the sign field is in no way constrained to only contain information on the sign of the signal, but can also capture morphological features, leading to a degeneracy between the two fields. This can be broken by either imposing constraints on the correlation structure for one or both of the fields or by introducing new data that informs the algorithm on one of the two fields. However, we find that the symmetry is sufficiently broken by the Gaussian process priors we imposed on both fields separately, in combination with the specific functional form of $\phi$ as given by Eq. (8). Sign variations can only be captured by the sign field, ensuring that it is structured. The overall amplitudes of these fluctuations, which change as a function of position on the sky, are more easily represented by the amplitude field, which therefore preferentially absorbs them. The fields are both assumed to have independent and Gaussian isotropic statistics. Their joint prior probability function is

$\mathcal{P}\left(\chi, \rho \mid S_{\chi}, S_{\rho}\right)=\mathcal{G}\left(\chi, S_{\chi}\right) \mathcal{G}\left(\rho, S_{\rho}\right)$.

In contrast to the distribution in Eq. (6), the covariances $S_{\chi}$ and $S_{\rho}$ are unknown. If this were not the case, the above model in a Bayesian setting would directly lead to a specific application of the well-known (non-linear) Wiener filter (Wiener 1966; Enßlin et al. 2009). This is not the case, unfortunately, as in our case we have little a priori knowledge on the correlation structure. Similar inference problems were solved in the previous reconstruction of the Faraday sky by NO12 and elsewhere (e.g., in Selig et al. 2015; Pumpe et al. 2018; Leike \& Enßlin 2019).

All of these inferences relied on a framework laid out by information field theory (IFT, Enßlin et al. 2009; Enßlin 2019). IFT connects Bayesian statistics with methods from statistical and quantum field theory, joining them into an inference scheme that connects noisy incomplete data with the underlying continuous field(s). Considering the problem mentioned above, the requirement of simultaneously inferring the map and the correlation structure of a field leads to the critical filter formalism, which was first formulated in IFT by Enßlin \& Weig (2010) and Enßlin \& Frommert (2011). We follow the most modern formulation, as outlined in Knollmüller et al. (2017). We summarize the modeling of the respective covariance matrices in the Appendix.

\subsubsection{The noise model}

We also need to find an estimate for the non-Galactic contributions to the Faraday rotation. In this part of the inference, we follow the approach in NO12 very closely. We start by inserting Eq. (3) into the measurement Eq. (4), and we define an effective noise term $\tilde{n}_{\phi}$ :

$d_{\phi}=\mathcal{R}_{\phi}\left(\phi_{\mathrm{gal}}+\phi_{\mathrm{etc}}\right)+n_{\phi} \equiv R_{\phi} \phi_{\mathrm{gal}}+\tilde{n}_{\phi}$ 
We have no reason to drop the assumption of Gaussianity for the new noise term $\tilde{n}_{\phi}$, but have to adapt the covariance to include the increase in uncertainty. As we have no a priori guesses on the specific systematics of the different sources, we infer this increase (or decrease) in uncertainty as well. This is implemented by introducing the following model,

$\widetilde{N}_{\phi}=\operatorname{diag}\left(\eta_{\phi} \sigma_{\phi}^{2}\right)$,

for the new noise covariance $\widetilde{N}$, where $\eta_{\phi}$ are the parameters that need to be inferred and $\sigma_{\phi}^{2}$ is the reported measurement uncertainty. We assume that $\eta_{\phi}$ follows an inverse gamma distribution:

$\mathcal{P}\left(\eta_{\phi} \mid \alpha_{\phi}, \beta_{\phi}\right)=\frac{\beta_{\phi}^{\alpha_{\phi}}}{\Gamma\left(\alpha_{\phi}\right)} \eta_{\phi}^{-\alpha_{\phi}-1} \exp \left(-\frac{\beta_{\phi}}{\eta_{\phi}}\right)$.

The hyper-parameters $\alpha_{\phi}$ and $\beta_{\phi}$ need to be specified for the inference. Conveniently, this model should also take care of sources suffering from $\mathrm{n} \pi$ ambiguities as an increased noise term down-weights the respective data points in the inference.

We chose the slope parameter for the power law to be $\alpha_{\phi}=2.5$. Furthermore, we imposed the prior expectation value of $\eta_{\phi}$ to be unity, which results in $\beta_{\phi}=1.5$. We note that this is a different choice of parameters from NO12 and NO15, where the authors chose $\alpha_{\phi}=1$ and $\beta_{\phi}$ such that the prior expectation value of $\ln \left(\eta_{\phi}\right)$ is zero. This implies that our values are more restrictive for the noise correction parameters and make it harder for the algorithm to classify data points as noise. This choice is motivated by the fact that we are now also inferring the profile field. In the old algorithm of NO12 the Faraday profile was fixed during the inference and was only determined after the inference of the sign field was performed. This made it easy for the algorithm to identify outliers; for example, cases where $\left|d_{\phi}\right| \gg|\phi|$ are only explainable by the noise correction if the signal field is already sufficiently determined at the location of the data point $d_{\phi}$. In our case the profile is a priori much more flexible and inferred simultaneously, making the identification of inconsistencies much more ambiguous. If we a priori want to have single data points to have a comparable impact to those in NO12, we have to restrict the noise estimation to some degree. We chose $\alpha_{\phi}$ and $\beta_{\phi}$ such that we were able to reproduce the previous results, as we have no reason to distrust the results of NO12 and NO15 and we have no better measure for the choice of hyper-parameters.

\subsection{Inference}

The hierarchical Bayesian model described in the previous sections can be symbolically depicted as a hierarchical tree, whose lower branches are shown in Fig. 4. The higher branches of the tree contain the hyper-parameters and are discussed in the appendix together with the more explicit likelihood and prior functions.

The evaluation of the posterior is a non-trivial task in our case, as the non-linearities in the models lead to highly nonGaussian distributions for which no analytical description is known to exist. Furthermore, the fact that we aim for a simultaneous inference of the fields determining the Faraday map, and their correlation structures, leads to a strong interdependence between all degrees of freedoms. Our desired resolution of $\approx 10$ arcmin also leads to a high dimensionality. Together with the high dimensionality of the problem, on the order of $10^{6}$ unknown parameters, this leads to considerable numerical complexity.

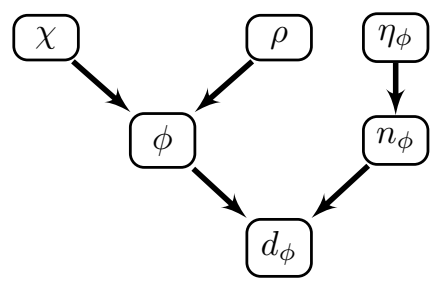

Fig. 4. Hierarchical Bayesian model for reconstruction II. We decompose the Faraday depth $\phi$ into the fields $\chi$ and $\rho$, which are supposed to capture the sign and the overall amplitude of the signal, respectively (see Eq. (8)). The Faraday depth field $\phi$ together with the measurement noise $n_{\phi}$ determine the observed data $d_{\phi}$ via Eq. (4). The noise $n_{\phi}$ of every measurement $i$ is assumed to be drawn from a Gaussian with variance $\eta_{i} \sigma_{i}^{2}$, where $\sigma_{i}$ is the reported uncertainty and $\eta_{i}$ an unknown uncertainty correction factor.

These problems can be solved via IFT, which provides efficient and well-tested algorithms for posterior evaluation. We employ here the Metric Gaussian Variational Bayes (MGVI) method, which is better suited for simultaneous map and correlation structure inference compared to the maximum a posteriori algorithm used in Oppermann et al. (2012). We furthermore implement the above-mentioned hierarchical tree as a forward model, which aims among other things for a more efficient numerical coupling between different parts of the model and allows the employment of sampling methods.

On the technical side, the inference was conducted using version 5 of the NIFTy python package (Selig et al. 2013; Steininger et al. 2017; NIFTy5 team et al., in prep.) for numerical information field theory. NIFTy is specifically designed for IFT applications and provides libraries for implementing the above mentioned models and for inferring their parameters and the uncertainties.

\subsection{Results}

\subsubsection{Comparison to older results}

The posterior mean Faraday depth map of reconstruction II relying on Faraday data only and inferred according to the model depicted in Fig. 4 is shown in Fig. 1b, the previous NO15 map in Fig. 1c, with corresponding standard deviations in Figs. 5b and $c$, respectively. The differences between the maps are shown in Fig. 2b. A visual comparison of the two maps reveals satisfying agreement. The difference map, however, shows that there are indeed significant small-scale deviations of the maps in the area of the Galactic disk. These may be a result of the different noise estimation parameters in the updated inference and/or the different treatment of the amplitude field (see discussion in Sect. 2.3.2). It should be noted that reconstruction II was conducted with a doubled resolution, which may also be responsible for some of the very small-scale differences.

The uncertainty maps nicely demonstrate the impact of the sky modeling on the inference. Figure $5 \mathrm{c}$ shows the uncertainty of the previous reconstruction, which exhibits imprints of the longitudinal Galactic profile used in the amplitude modeling. Our updated uncertainty map in Fig. 5b shows a decrease in uncertainty in certain regions, especially in the Galactic disk. This simply reflects that our updated inference scheme can use the correlation information encoded in the data much more efficiently.

The power spectra depicted in Fig. 6 also need to be inspected as they have been inferred with different algorithms in NO15 and the approach chosen in reconstruction II. We note 


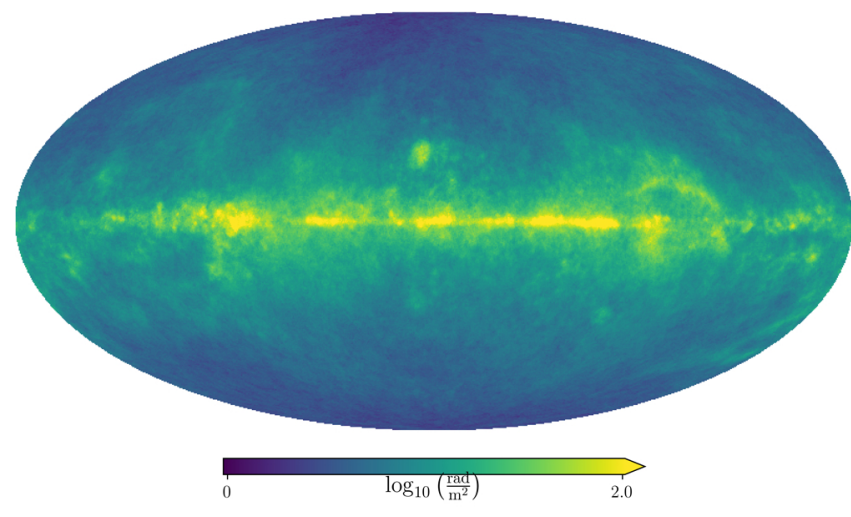

(a)

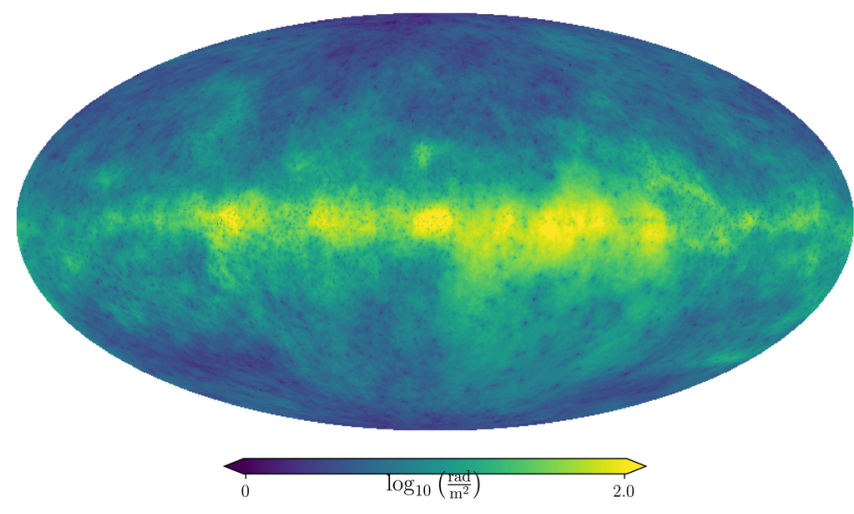

(b)

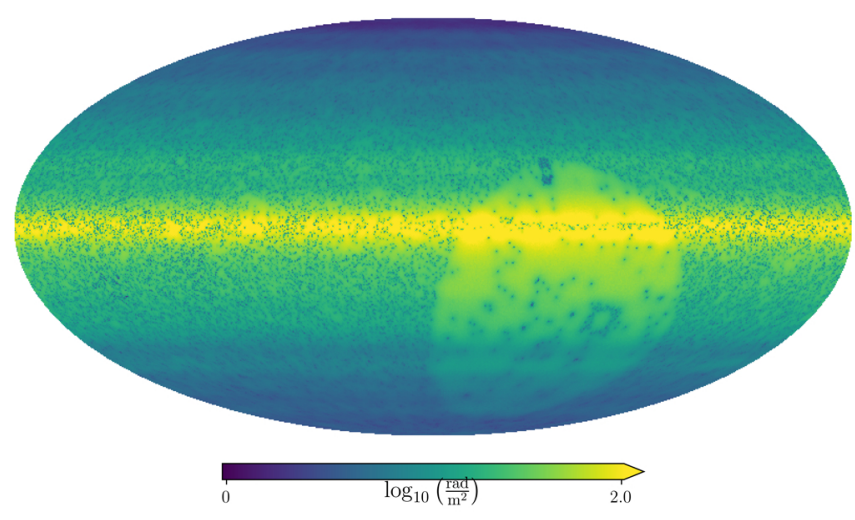

(c)

Fig. 5. Logarithmic posterior uncertainties of the different reconstructions. Panel $a$ : posterior uncertainty in $\phi$ of reconstruction I (whose mean is shown in Fig. 1a). Panel $b$ : posterior uncertainty corresponding to reconstruction II (shown in Fig. 1b). Panel c: posterior uncertainty corresponding to the NO15 reconstruction (shown in Fig. 1c).

that we can confirm the slope of the spectrum. The revised map resulting from reconstruction II has significantly less power on small scales. Again, the parameter change in the noise estimation may be the reason for this difference, as we know that it can have a considerable impact on smaller scales, as we change the ability of the algorithm to increase uncertainties to accommodate outliers in the data.

With respect to the error estimation, we show in Fig. 7 the comparison of the reported observational noise standard

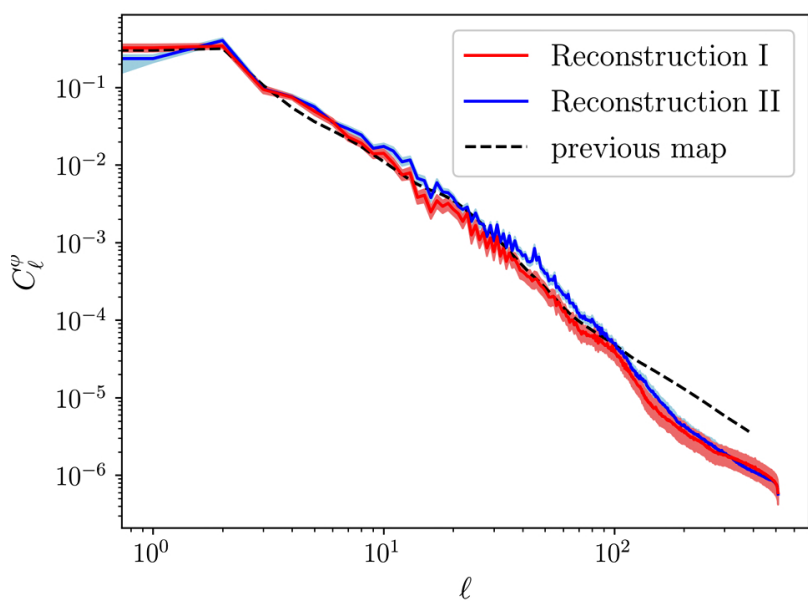

Fig. 6. Comparison of the power spectrum inferred by NO15 (dashed black) to the spectrum of the Faraday map including the free-free EM data (reconstruction I, red) and the results of reconstruction II in Sect. 2 (blue). The latter two inference algorithms also produce uncertainties in the power spectrum, depicted as shaded areas in the respective colors.

deviation $\sigma$ and our estimate for the noise $\widetilde{\sigma}$ according to Eq. (10). The uncertainties of some sources have been multiplied by factors of up to 1000 , indicating strong internal or extraGalactic contributions to these particular Faraday data points. Nonetheless, we confirm or even decrease the uncertainties for most measurements, as demonstrated by the contour lines shown in the figure, which represent a normalized density plot in the $\sigma-\widetilde{\sigma}$ plane. We furthermore highlighted the nine data points that were identified by Ma et al. (2019) as $\mathrm{n} \pi$-ambigious. Our algorithm has down-weighted all of them, indicating its capability of identifying problematic data. The noise estimation results for the subset of the Taylor catalog analyzed by Vernstrom et al. (2019) is shown in Fig. 7b. A large fraction of the error bars of these data points were decreased, in accordance with Vernstrom et al. (2019), who draw their conclusions from a reanalysis of the original polarimetric data.

With this we conclude the comparison between the NO15 results and our reconstruction II, and turn to the analysis of the underlying components of our sky model defined in Eq. (8).

\subsubsection{Sign and amplitude fields}

The sign and amplitude fields $\chi$ and $\rho$ are shown in Figs. 8b and $9 \mathrm{~b}$, respectively. The sign field captured most of the smallscale structure, but has almost completely lost all information on the Galactic disk profile, which was absorbed by the amplitude field, as intended by our modeling. The field $\chi$ shows some similarity to the corresponding signal field inferred by NO15, which is shown in Fig. 8c. The exponentiated amplitude field appears to be relatively smooth with few distinguished features apart from the large-scale disk profile. These features, however, show remarkable similarity to the Galactic EM as traced by free-free emission, which was precisely mapped by missions investigating the cosmic microwave background (CMB) such as Planck (Planck Collaboration X 2016), as it is a important foreground component in the microwave sky. We show the Planck free-free EM map in Fig. 9a. The color scales were chosen to highlight the structures in the maps that we think originate from the same Galactic structures. These apparent resemblances (which can already be seen by simply taking the absolute value of the NO15 map) between the two maps have motivated us to 


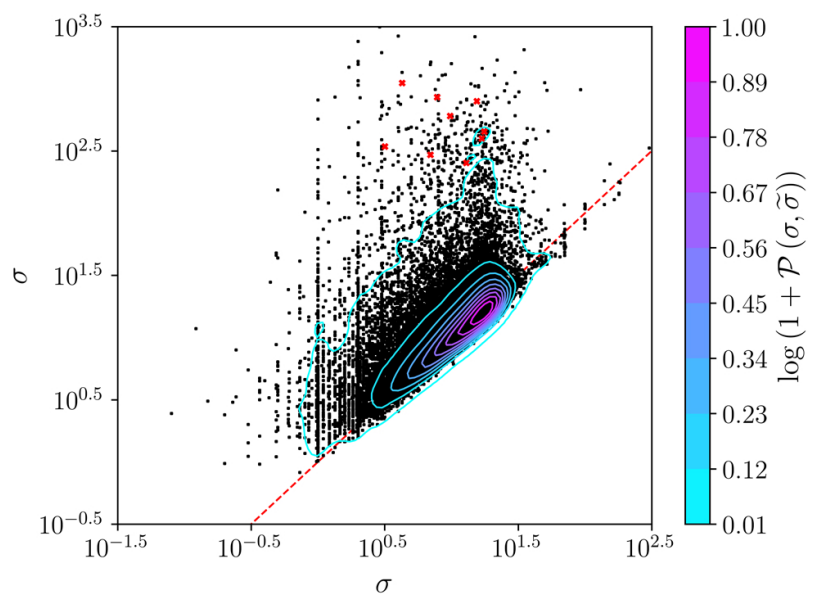

(a)

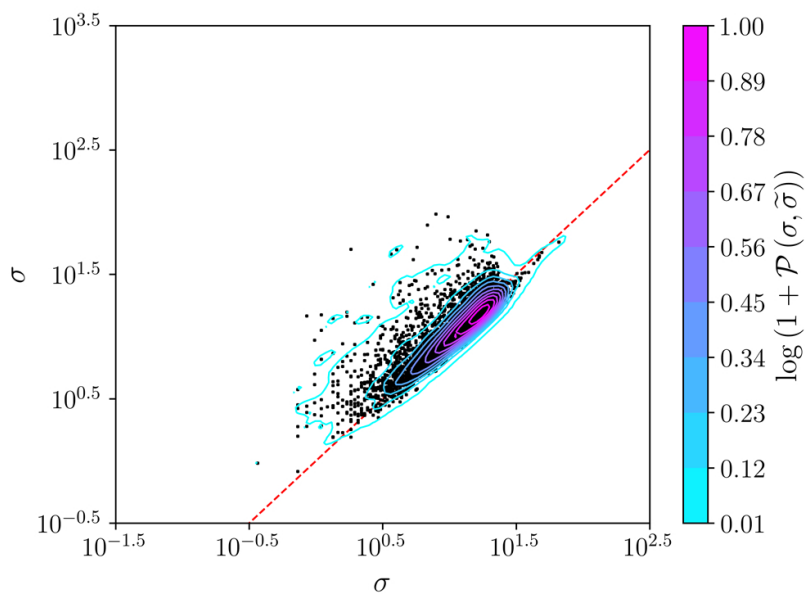

(b)

Fig. 7. Estimated noise standard deviations $\widetilde{\sigma}$ compared to the $\sigma$ values given by the Oppermann catalog and for the subset analyzed by Vernstrom et al. (2019). To counteract the visual impression which is dominated by the outliers, we overlaid contour lines from the underlying log probability density function estimated via Gaussian kernels. Equality between the two quantities is indicated by the red dashed line. The red crosses indicate the data points identified by Ma et al. (2019) as $\mathrm{n} \pi$-ambigious.

include the free-free EM map in our inference. An investigation of the physical plausibility of this correlation (and its absence for some regions) is given in the next section.

\section{Including the free-free emission}

\subsection{The physics}

In addition to the visual similarities between the free-free EM map and our inferred amplitude field, we wanted to further motivate this apparent congruence by investigating the physics of the free-free emission and the Faraday rotation a bit more closely. The EM traced by the bremsstrahlung emitted by thermal electrons on protons in the warm interstellar medium can be quantified via (see, e.g., Smoot 1998)

$\operatorname{EM}_{\mathrm{ff}}\left(n_{\mathrm{th}}\right)=\int_{\mathrm{LOS}} \mathrm{d} l n_{\mathrm{th}}^{2} \equiv \int_{\mathrm{LOS}} \mathrm{d} l \mathrm{em}$ ff

where we defined $\mathrm{em}_{\mathrm{ff}} \equiv n_{\mathrm{th}}^{2}$ as a source term for $\mathrm{EM}_{\mathrm{ff}}$.

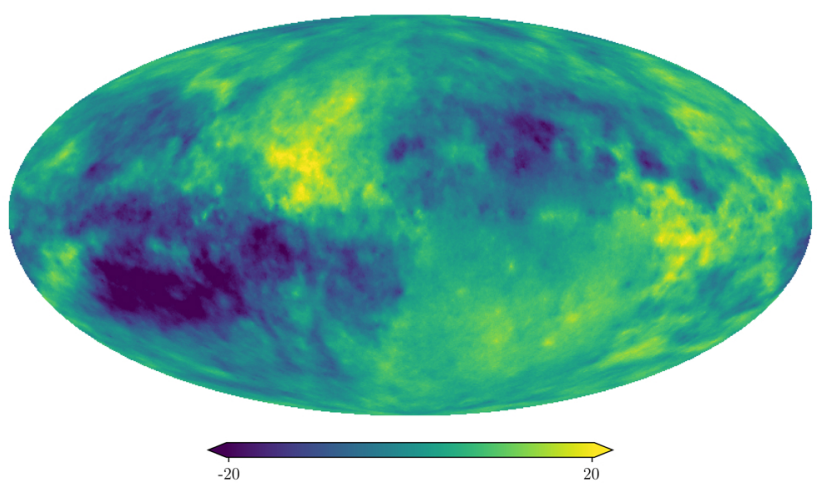

(a)

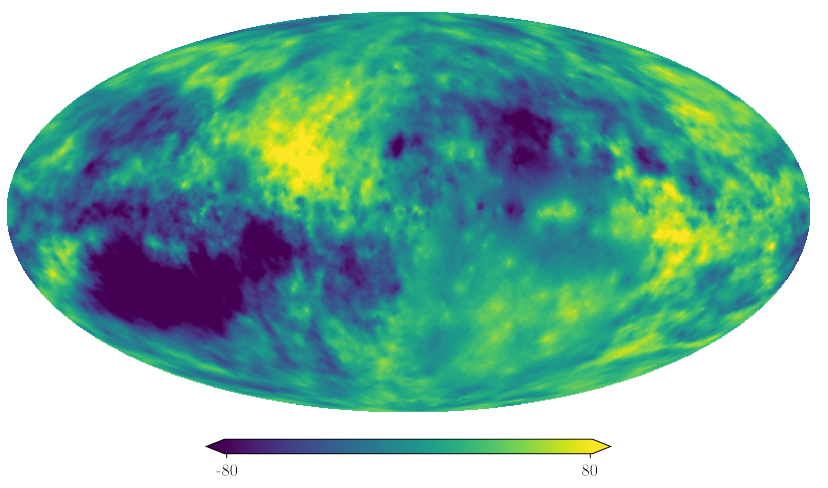

(b)

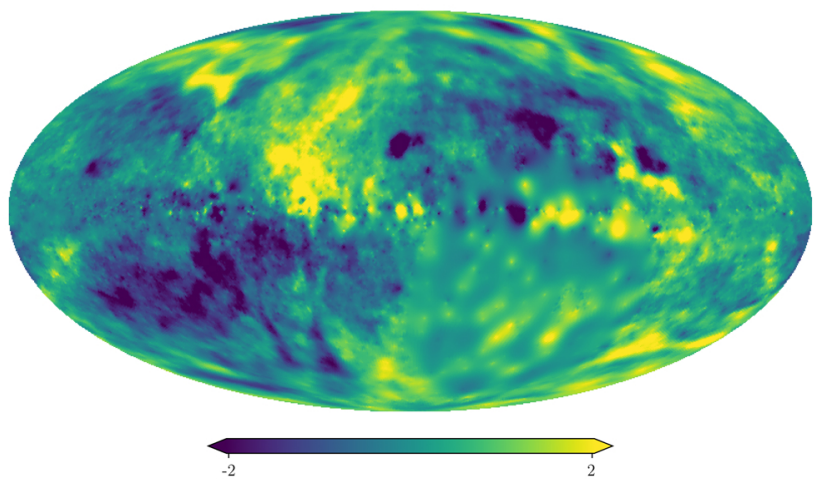

(c)

Fig. 8. Sign fields of the different reconstructions. Their primary purpose is to capture the sign variations of the Faraday sky. Panel $a$ : field $\chi$ of reconstruction I, as defined by the model in Eq. (20). Panel $b$ : field $\chi$ of reconstruction II, as defined by the model in Eq. (8). Panel $c$ : field $s$ of the previous reconstruction by NO15, which served a similar purpose. The difference in the scales of these fields is compensated by corresponding differences in the respective Faraday amplitude fields.

If we turn to the Faraday depth and want to study Eq. (2) with the intention of a comparison to $\mathrm{EM}_{\mathrm{ff}}$, we must quantify the dependence of $B_{\mathrm{LOS}}$ on the thermal electron density. In general, we can assume the magnetic flux to be frozen into the Galactic interstellar medium (ISM; de Gouveia Dal Pino 2006). The exact nature of the $B-n_{\text {th }}$ relationship, however, is complicated as it strongly depends on the morphology and dynamics of the plasma under consideration. In the simplest case of an isotropically collapsing structure, flux freezing leads to a 
$\operatorname{EM}\left[\frac{\mathrm{pc}}{\mathrm{cm}^{6}}\right]$

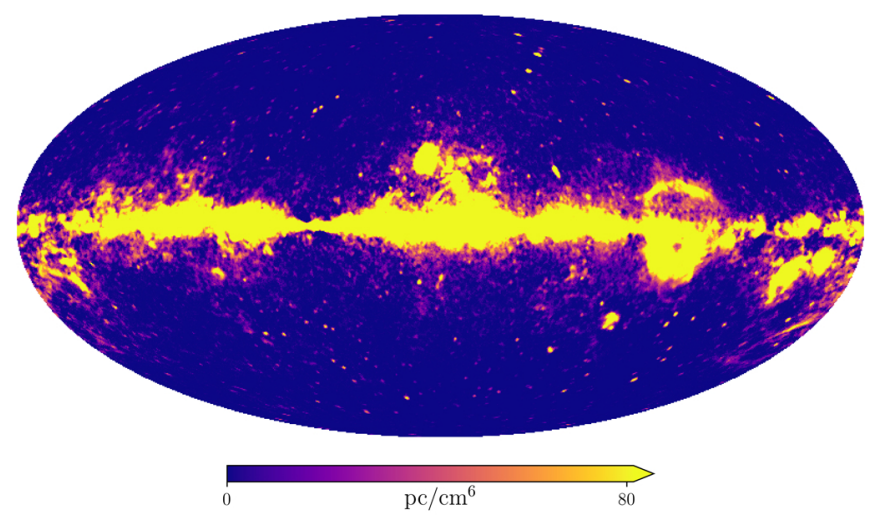

(a)

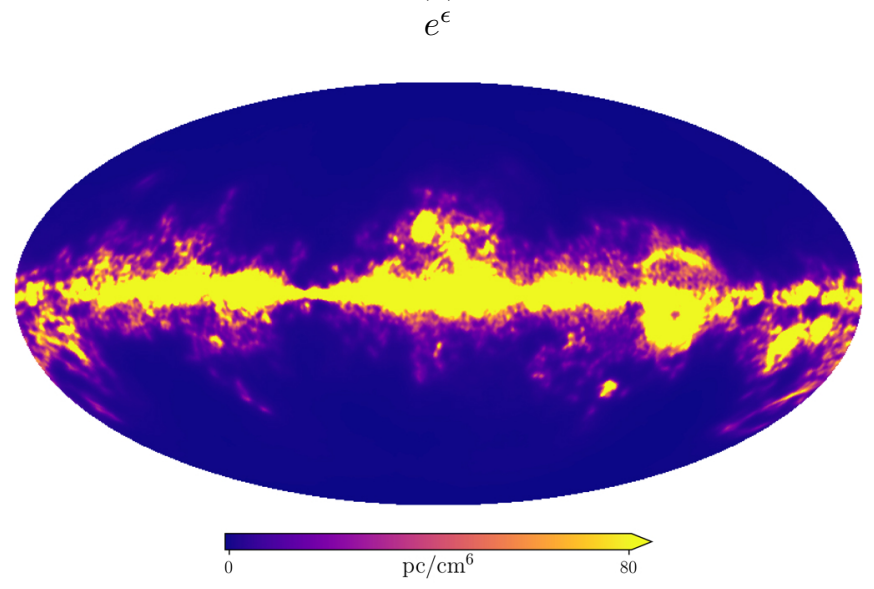

(c)

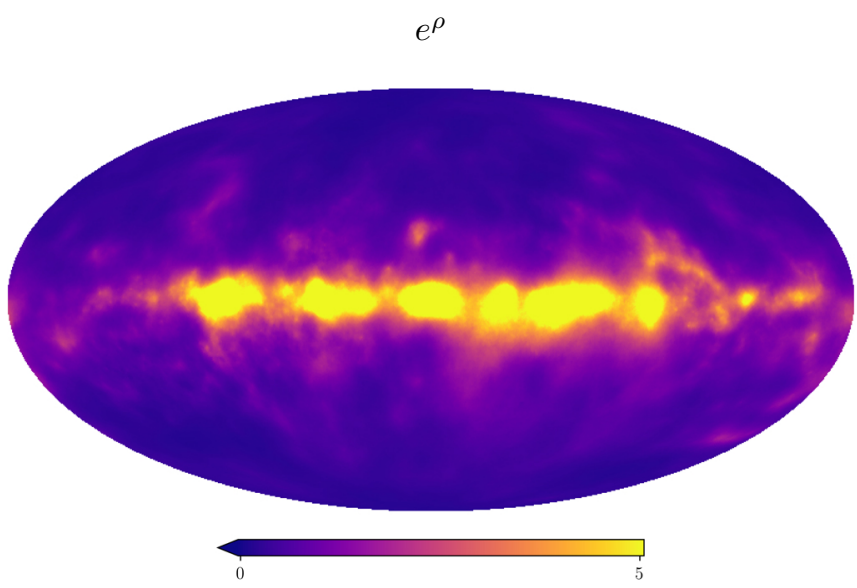

(b)

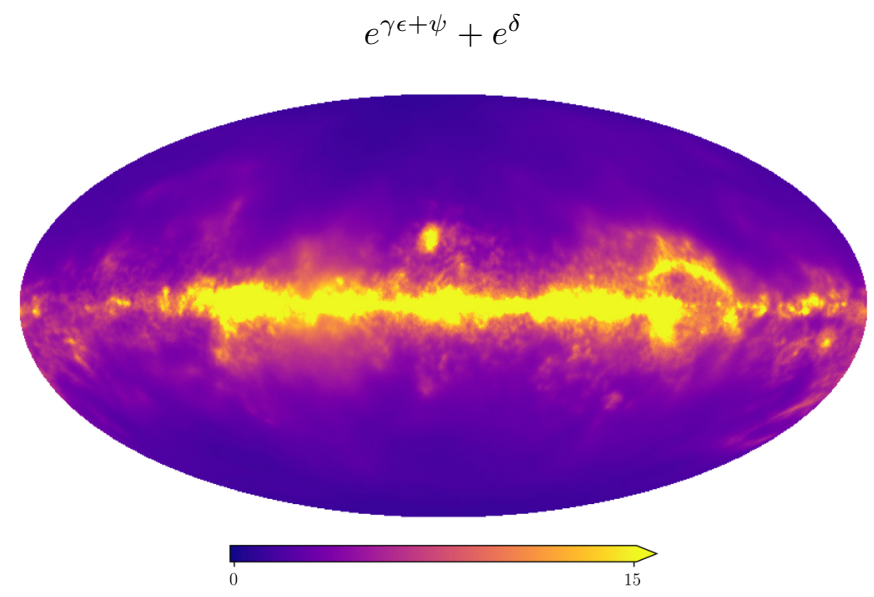

(d)

Fig. 9. Panel a: Galactic free-free EM map obtained by the Planck Collaboration (Planck Collaboration X 2016). The other figures show the amplitude fields of the different reconstructions, which were supposed to capture the amplitude variations of the Faraday sky. Panel $b$ : exponentiated amplitude field $\rho$ of the reconstruction II, defined in Eq. (8). This field is not constrained by free-free data. Panel $c$ : exponentiated $\epsilon$ field defined in Eq. (18). This field is part of the revised Faraday map resulting from reconstruction I and the denoised free-free sky. A logarithmic version of this plot is shown in Fig. 10a. Panel d: full Faraday amplitude field of reconstruction I, defined in Eq. (20). This field is part of the result of the model in Eq. (20). A logarithmic version of this plot is shown in Fig. 10d.

$|B| \propto n_{\text {th }}^{\frac{2}{3}}$ dependence (Mestel 1966). For a realistic description of the ISM, however, we need to consider non-linear magnetohydrodynamics (MHD). Under certain simplifying assumptions, as shown in Passot \& Vázquez-Semadeni (2003), it is possible to decompose the ISM flow into several wave types corresponding to different modes, leading to different density dependences of the magnetic field strength. Most notably in the low density regime, the dominating mode approaches a constant in $n_{\mathrm{th}}$, implying no correlation between $|B|$ and $n_{\text {th }}$ in this regime. Furthermore, as waves corresponding to different modes may very well travel through the same regions within a short time span, the $B-n_{\text {th }}$ correlation may be subject to large variability in all regimes of measured electron density (Vázquez-Semadeni 2015). This pattern was confirmed for tracers of non-ionized hydrogen such as Zeeman splitting by Crutcher et al. (2010), among others, who found no $B-n_{\mathrm{H}}$ correlation for particle density regimes $n<300 \mathrm{~cm}^{-3}$, and strong variations in the regime above in molecular clouds, indicating a power-law dependence of roughly $|B| \propto n_{\mathrm{H}}^{0.65}$. Furthermore, Harvey-Smith et al. (2011) found no correlation between $n_{\text {th }}$ and $|B|$ in several known
HII regions, nor did Purcell et al. (2015) specifically for the Gum nebula.

We therefore assume the dependence of the magnetic field strength on $n_{\text {th }}$ to follow a power law with an unknown and spatially dependent coefficient $|B| \propto n_{\text {th }}^{p_{B}}$, which could very well be zero. We can then rewrite the proportionality of the absolute value of the integrand of the Faraday depth in Eq. (2) on $n_{\mathrm{th}}$ :

$$
\begin{aligned}
B_{\mathrm{LOS}} n_{\mathrm{th}} & =|B| \cos (\theta) n_{\mathrm{th}} \\
& \propto n_{\mathrm{th}}^{1+p_{B}} \cos (\theta) \propto \mathrm{em}_{\mathrm{ff}}^{\frac{1+p_{B}}{2}} \cos (\theta) .
\end{aligned}
$$

Here we introduced the angle $\theta$ indicating the orientation of the magnetic field with respect to the LOS. Its impact is discussed below. If $p_{B} \approx 1$ for all locations along a line of sight, the absolute value of the Faraday integrand strongly depends on the free-free emissivity, and the additional electron density dependence is completely canceled. In the unrealistic case that $p_{B}=-1$, the absolute value of the Faraday integrand would be completely uncorrelated to the free-free source term. As discussed before, in some cases the electron density is not a good 
tracer for the magnetic field strength and the LOS projection might partly mask the relation in the observables, which again limits the above relationship. Nonetheless, we think that the above considerations motivate the inclusion of the free-free map as a proxy for the Faraday amplitude field in our inference, as most realistic cases of the power-law index $p_{B}$ will result in a correlation between the free-free EM and the Faraday sky. On the other hand, this analysis also indicates that the effects of ISM dynamics on the magnetic field strength have to be considered for a reliable inference.

A second effect that needs to be taken into account are sign reversals of the magnetic field along the line of sight, which could strongly affect the value of $\phi$ in contrast to $\mathrm{EM}_{\mathrm{ff}}$. To consider these factors, we approximate the line of sight integral via

$$
\begin{aligned}
\phi & \propto \int_{\mathrm{LOS}} \mathrm{d} l|B| \cos (\theta) n_{\mathrm{th}}=L\left\langle|B| n_{\mathrm{th}} \cos (\theta)\right\rangle_{L} \\
& \approx L^{2}\left\langle|B| n_{\mathrm{th}}\right\rangle_{L}\langle\cos (\theta)\rangle_{L}
\end{aligned}
$$

where $L$ is the length of the line of sight and $\langle\ldots\rangle_{L} \equiv \frac{1}{L} \int_{\operatorname{LOS}} \mathrm{d} l \ldots$ indicates an averaging process along the line of sight. The last approximation implies statistical independence between the magnetic field strength and $\theta$. This shows that, to a good approximation, the geometry of the magnetic field can lead to a multiplicative term, which is not constrained by the free-free data alone and has to be considered independently. In our setup, it can only be determined by discrepancies between the Faraday and free-free data sets. Both effects, the unknown $B-n_{\text {th }}$ correlation and the potential sign reversals, might be responsible for discrepancies between the free-free data and the Faraday amplitudes resulting from our reconstruction II (see Fig. 9b).

As in the first part of this paper, we first discuss properties of the EM data and then show the modeling of the respective sky maps in Sect. 3.3.

\subsection{Data}

In this work we use the free-free EM map of the Planck 2015 release (Planck Collaboration X 2016). This map is a side product of a foreground subtraction process of the Planck team in their pursuit of the CMB sky reconstruction. The freefree sky is a dominant component of this foreground in the low frequency regime, along with synchrotron and spinning dust emission (Planck Collaboration XXV 2016). Most components in the microwave sky are hardly distinguishable as they result from similar structures in the sky. Planck therefore employs a sophisticated component separation algorithm called COMMANDER (Eriksen et al. 2008), mostly exploiting the different energy spectra of the components. This algorithm uses the Gibbs sampling technique to approximate the posterior distribution for all components, also making use of the WMAP data (Bennett et al. 2013) and a $408 \mathrm{MHz}$ survey map (Haslam et al. 1982; Remazeilles et al. 2015) for the separation. An updated version of COMMANDER employed in the 2018 release of Planck takes the correlation structure of the components into account. However, Planck did not release an updated free-free map in 2018.

It should be noted that the measured free-free EM also contains a dependency on the thermal electron temperature $T_{\mathrm{e}}$. The Planck team has modeled the free-free emission as an analytical combination of both EM and $T_{\mathrm{e}}$, which produces separate maps for the two quantities. This is a considerable advantage of the Planck data over other tracers of EM, such as H- $\alpha$ data, for which full sky maps also exist, but for which temperature and EM have not been separated. As we have no good model of the dependence of the magnetic field and consequently of the Faraday depth on $T_{\mathrm{e}}$, we prefer to use the pure EM data as this minimizes the physical systematics that have to be modeled.

As a side product of the estimation of the posterior via sampling, Planck provides us not only with the posterior mean of the free-free component, but also with uncertainties, which are a valuable source of information and lets us treat the free-free data as a slightly noisy measurement of the true free-free EM with known uncertainties. Unfortunately, upon inspecting the Planck data, we found some pixels to have extremely small variances, and in case of the free-free temperatures even zero variances. This is rather surprising given the large degeneracy between the components, and we expect this to be a numerical artifact of the sampling procedure or of the marginalization process that results in the uncertainties for specific components. Our algorithm relies on accurate uncertainty measures for all pixels. In the case of a severe underestimation of the errors in some specific area, this region is over-weighted by the algorithm in the inference of the correlation structure, thereby immediately affecting other parts of the sky and subsequently all other maps of the inference. This has to be accounted for if one is not completely sure that the noise values are free of systematics. We therefore perform a noise estimation procedure similar to that done for the Faraday data.

With this in mind, we write the measurement equation for the free-free EM data as

$d_{\mathrm{ff}}=\mathcal{R}_{\mathrm{ff}} \mathrm{EM}_{\mathrm{ff}}+n_{\mathrm{ff}, \mathrm{obs}}+n_{\mathrm{ff}, \mathrm{sys}} \equiv \mathcal{R}_{\mathrm{ff}} \mathrm{EM}_{\mathrm{ff}}+\widetilde{n}_{\mathrm{ff}}$.

In the case of Planck, the response $\mathcal{R}_{\mathrm{ff}}$ is just a unit matrix, as the data is already provided as a full sky map on the resolution we desire. Here we decompose the noise $n_{\mathrm{ff}}$ into two components, namely an observed one obtained by COMMANDER and an unobserved one, which contains systematics. As we do not consider the correlation structure of the noise for any data set, hereafter the procedure of noise estimation is the same as in Sect. 2.3.2, apart from different hyper-priors. In this case, we choose $\alpha_{\mathrm{ff}}=1$ and $\beta_{\mathrm{ff}}$ such that the expectation value over $\ln \left(\eta_{\mathrm{ff}}\right)$ is zero, as we a priori expect most of the noise values to be correct, but the $\eta_{\mathrm{ff}}$ parameter to vary over orders of magnitude in the cases when they are not.

The lower branches of the free-free sky model are shown in Fig. 11. The full extended model for the hierarchical Bayesian model is shown in Fig. 12. Using the considerations above, the likelihood for the free-free sky is again Gaussian in analogy to Eq. (7),

$\mathcal{P}\left(d_{\mathrm{ff}}\right)=\mathcal{G}\left(d_{\mathrm{ff}}-\mathcal{R}_{\mathrm{ff}} \mathrm{EM}_{\mathrm{ff}} \mid \widetilde{N}_{\mathrm{ff}}\right)$,

with the noise covariance $\widetilde{N}_{\text {ff }}$ defined in analogy to Eq. (10). Again, we still need to explain the detailed model for $\mathrm{EM}_{\mathrm{ff}}$ and its priors. This is presented in the next section.

\subsection{The sky model}

We now turn to the modeling of the free-free sky and its connection to the amplitude field of the Faraday sky under the constraint of the physical limitations considered in Sect. 3.1. We model $\mathrm{EM}_{\mathrm{ff}}$ with a log-normal approach, again to enforce positivity and to capture the expected large variability of $\mathrm{EM}_{\mathrm{ff}}$ on the sky:

$\mathrm{EM}_{\mathrm{ff}} \equiv e^{\epsilon} \cdot \frac{\mathrm{pc}}{\mathrm{cm}^{6}}$ 
If the true free-free EM sky were a perfect estimator for the amplitude field of the Faraday sky, we could simply replace $\rho$ in Eq. (8) with $\epsilon$ to simultaneously infer both quantities from the two data sets. However, as discussed in Sect. 3.1, a necessary condition for a combination of the information of free-free $\mathrm{EM}$ and Faraday rotation measures is to account for magnetic field reversals, different scalings with the thermal electron density, and potential systematics in the data sets. All issues can be accounted for by introducing further terms to the model.

We start with our approach to model the differences in the electron density scaling and the geometry factor introduced by the sign reversals. As we can only work with the LOS integrated quantities, the most natural way of inferring the 3D effective scaling factor $\frac{1+p_{B}}{2}$ in Eq. (14) is not possible, as this would necessarily imply setting up the full 3D problem for which we lack the necessary depth information. In the future this information might be provided by a sufficient number of rotation measures and dispersion measures (DMs) from pulsars, which was demonstrated with mock data for DMs by Greiner et al. (2016). We therefore choose to introduce two scaling fields $\gamma: S^{2} \rightarrow \mathbb{R}$ and $\psi: S^{2} \rightarrow \mathbb{R}$ into the modeling according to Eq. (19). This essentially sets up a linear fit for the logarithm of the Faraday amplitude field in terms of the reconstructed logarithmic free-free sky:

$\phi=\left(e^{\gamma \epsilon+\psi}\right) \chi$.

This model somewhat mimics the approximation derived in Eq. (15), with $\gamma$ approximating the role of the power-law coefficients and $e^{\psi}$ the geometry factor. Unfortunately, we lose the interpretation of the $\gamma$ field in terms of $\frac{1+p_{B}}{2}$ in Eq. (14) due to the above-mentioned lack of consistent 3D modeling. Furthermore, the large-scale modes of the $\psi$ and $\gamma$ fields are to a degree degenerated, making a quantitative interpretation of the $\psi$ field very hard, although the smaller scale morphology may be informative. Altogether, these terms should be able to capture sign reversal effects and strong deviations from the proportionality of the magnetic field strength on the thermal electron density presented in Eq. (14).

We also have to consider the case where the Faraday sky contains amplitude structure not present in the $\mathrm{EM}_{\mathrm{ff}} \mathrm{sky}$. This is done by expanding the model according to

$\phi=\left(e^{\gamma \epsilon+\psi}+e^{\delta}\right) \chi$.

Here the second amplitude field $\delta: S^{2} \rightarrow \mathbb{R}$ is not connected to the free-free EM map, giving it the possibility to capture structures that are in the Faraday data but not in the free-free data. All newly introduced fields are assumed to follow Gaussian statistics, so their prior is similar to Eq. (9), again with unknown correlation structure. It should be noted at this point that the obvious a priori degeneracies between the newly introduced helper fields are usually broken during the inference, if sufficiently supported by data. This is possible due to the joint correlation structure inference, which constrains the respective fields just to the degree it is demanded by the inference problem.

Although there is motivation for each term, the above modeling might be viewed as ad hoc, as it is certainly possible to argue for the addition or omission of certain terms or a completely different modeling altogether. There are certain limitations for potential models, as they all have to be able to capture the physical characteristics (e.g., the sign reversal of the Faraday map), and should be kept as simple as possible.
Unfortunately, these requirements already allow for a range of different parametrizations. We have varied the model during our analysis by adding or omitting further terms in Eq. (20), for example. We found little variation in the overall morphology of the Faraday sky, apart from the disk, where omission of the $\gamma$ and $\psi$ field lead to extremely pronounced features, with Faraday depth values reaching regimes a magnitude higher than previous reconstructions. As we think that these physical considerations are too important to be neglected, we deem the resulting maps in these cases to be unrealistic. Given that all newly introduced helper fields have captured distinct structures, as we will show in Sect. 3.4, we view the model in Eq. (20) as one of the simplest cases that can capture all relevant effects, but nonetheless we deem the systematic errors higher than the statistical ones in this inference.

To gain some insights on the robustness of the obtained freefree EM map, we also performed an inference just for the $\mathrm{EM}_{\mathrm{ff}}$ sky with the model in Eq. (18), unconstrained by Faraday data. The comparison of the result to the outcome of the joint Faraday and $\mathrm{EM}_{\mathrm{ff}}$ inference is presented in the results section. We can implement this extended model numerically with similar algorithms, as described in Sect. 2.4.

\subsection{Results}

\subsubsection{The revised Faraday map}

In the following we show the results of the model depicted in Fig. 12. We show the mean, the differences from the results of NO15 and reconstruction II, and the power spectrum in Figs. 1a, 2a,c, and 6. The inclusion of the free-free sky has lead to a notable difference in our newly revised estimate of the Faraday sky. Driven by the strong disk in the free-free data, the Faraday sky now has a similarly strongly pronounced disk feature as well. Other features, mostly farther away from the Galactic plane, remained relatively stable, such as the northern arc of the Gum nebula, which is also strongly visible in the free-free data. In Fig. 5a, we show the updated uncertainties. These have again narrowed considerably compared to reconstruction II, as shown in Fig. 5b. The innermost part of the disk, however, is still quite uncertain. We also note that model uncertainties are not considered in this plot. The region of increased uncertainty in the southern sky is no longer discernible. This effect can be attributed to the inclusion of the EM data, which now strongly constrains the profile field in this area. The new power spectrum of the Faraday map is shown as the red line in Fig. 6. It is very similar to that of reconstruction II, with a notable offset towards smaller scales, implying a slightly steeper power law.

\subsubsection{Components}

We now discuss some of the components of the Faraday model in Eq. (20), apart from the $\epsilon$ field in Fig. 10a, which is more closely debated in the following section dedicated to the inference results of the free-free EM sky.

The revised Faraday amplitude is shown in Fig. 9d. The comparison to the amplitude field of reconstruction II in Fig. 9b and the free-free data in Fig. 9a demonstrate the influence of both the Faraday and the free-free data on the new field. The field is enhanced by roughly a factor of 4 compared to the old amplitude field. This may be a result of the symmetry-breaking process described in Sect. 2.3, as the amplitude field is now partially constrained by the free-free sky. A comparison of the sign fields demonstrates that this factor was mostly absorbed there. 
$\epsilon$

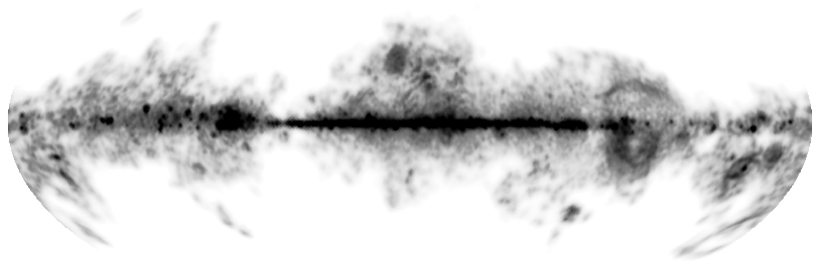

(a)

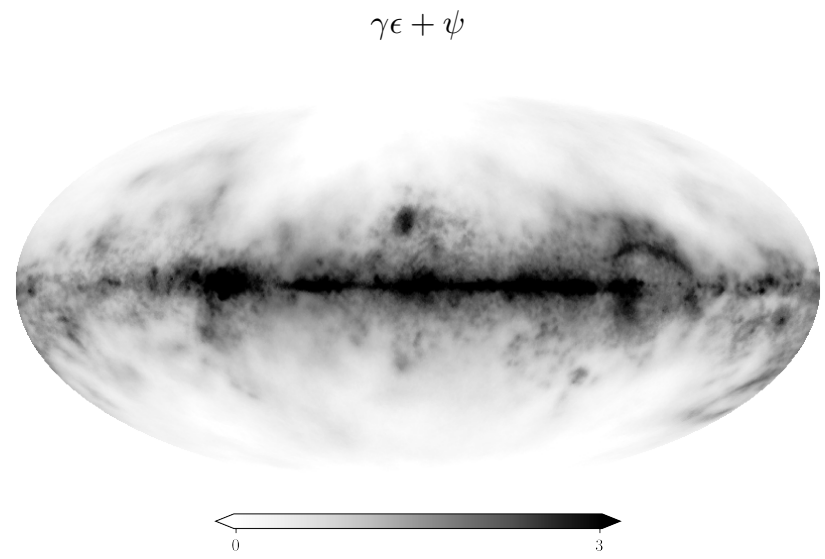

(c) $\delta$

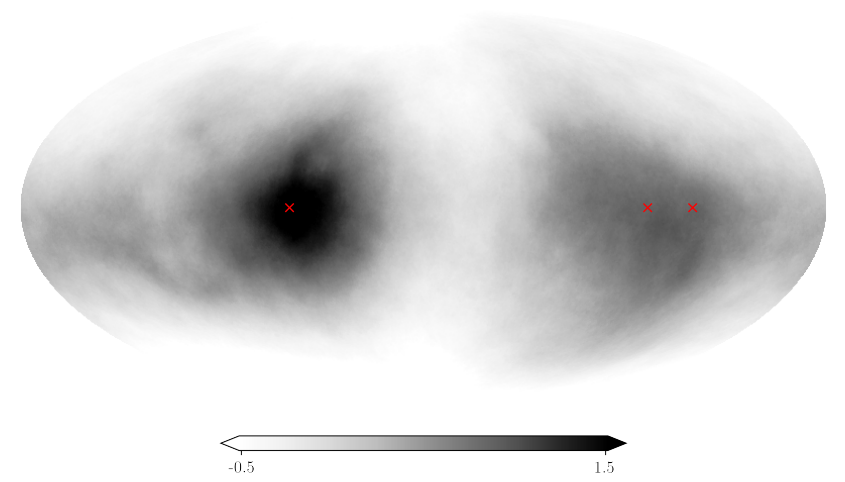

(b)
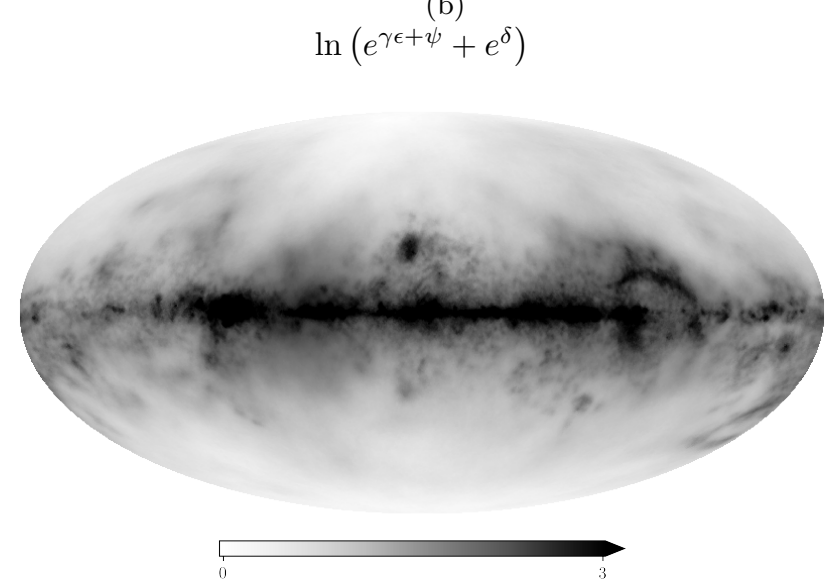

(d)

Fig. 10. Logarithmic amplitude field of reconstruction I, as defined in Eq. (20), and its components. Panel $a$ : field $\epsilon$ defined in Eqs. (20) and (18), constrained by free-free and Faraday data. This is also the reconstructed log free-free map. Panel $b$ : second amplitude field $\delta$ defined in Eq. (20). The red crosses indicate the approximate angular positions of the Orion arm, as given by Vázquez et al. (2008) and Xu et al. (2009). Panel c: logarithmic amplitude of the Faraday sky, as defined in Eq. (19) without the additional $\delta$ contribution, while it is included in $d$.

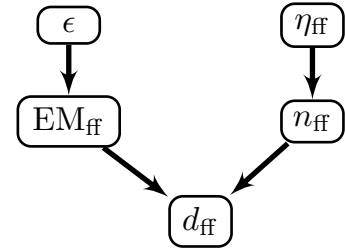

Fig. 11. Hierarchical tree model for the free-free EM sky. We model $\mathrm{EM}_{\mathrm{ff}}$ via the exponentiated field $\epsilon$ (see Eq. (18)). The field $\mathrm{EM}_{\mathrm{ff}}$ is, together with the noise $n_{\mathrm{ff}}$, connected to the observed data via Eq. (16).

The second amplitude field $\delta$ is shown in Fig. 10b, its influence on the total logarithmic Faraday amplitude is shown in Figs. 10c and d. The field is dominated by two diffuse structures of enhanced $\delta$, centered on the Galactic plane and separated by roughly $180^{\circ}$ longitude. Remembering the discussion in Sect. 3.1, the $\delta$ field is supposed to predominately capture amplitude variations between the free-free and the Faraday sky. Higher values of $\delta$ can be caused by systematic errors in either the EM or the Faraday data and/or a high alignment of the magnetic field with the LOS in this direction. The approximated longitudinal difference of the two $\delta$ enhancements of about $180^{\circ}$, as well as their positions, seem to correspond to the angular positions of the Orion arm on the sky, which are depicted in the plot as red crosses. In the first quadrant of the Milky Way, at about $60^{\circ}$ longitude, this minor arm is known to extend over several kiloparsec up to the Sagittarius arm, where it might merge (Xu et al. 2009). In the other direction towards the third quadrant of the Galaxy, the structure of the arm is much more complex. Even a bifurcation or a crossing with the Perseus arm seems possible (Vázquez et al. 2008). A overview of the properties of the Local Arm is given by (Vallée 2018). Moreover, the Local Arm can also be localized in 3D reconstructions of the local dust distribution (see, e.g., Lallement et al. 2018; Leike \& Enßlin 2019). We have indicated possible continuations of the arm in the plot at $240^{\circ}$ and $260^{\circ}$ longitude, respectively. The $\delta$ field seems to reflect not only the position of the arm, but also its morphology, as the structure in the third quadrant is more diffuse, as opposed to the relatively pronounced structure at $60^{\circ}$ longitude. All in all, this would argue for a high magnetic field alignment as a viable possibility for the interpretation of the second amplitude field. As the Sun is positioned directly in the Orion arm, we expect more coherent magnetic fields along the LOS directed pointed directly to the dominant direction of the arm. Remembering the discussion in Sect. 3.1, this then may lead to a disproportionate increase in the observed Faraday data as the free-free EM map would indicate at these positions. Furthermore, the Faraday 


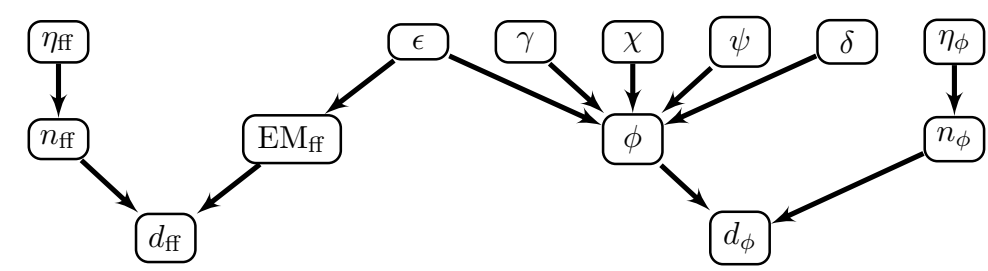

Fig. 12. Full hierarchical model excluding the power spectrum hyper-priors for the fields. The lowest layer contains the data sets $d_{\mathrm{ff}}$ and $d_{\phi}$, which are connected by Eqs. (4) and (16) to the sky maps $\mathrm{EM}_{\mathrm{ff}}$ and $\phi$. The sky maps in turn are connected to the Gaussian fields in the uppermost layer via the respective models defined in Eqs. (18) and (20). From there on, the higher branches for the respective correlation structure inference follow. They are not depicted here, but are shown and explained in the appendix.

sky in Fig. 1a indeed shows opposite magnetic field signs for those regions, as would be expected for a field traversing the solar location. We show the field

$\phi_{\text {Orion }}=e^{\delta} \chi$,

in Fig. 13a, which corresponds to our estimate of the enhancement of the Orion Faraday depth, if the above considerations were true and if the $\chi$ field really only captured the sign. The complementary Faraday component (in terms of amplitude contributions) is shown in Fig. 13b. The considerable differences with Fig. 1a in the sky regions corresponding to the Orion arm reveal again the influence of the $\delta$ field there. Of course we cannot rule out any of the other explanations for the observed $\delta$ enhancements, and the plot in Fig. 13a should therefore be taken with care. On the other hand, we can find additional evidence for magnetic field LOS alignment in the Orion Arm and the adjacent sign reversal region and for a physical counterpart of the $\delta$ field in the literature. For example, the enhancement in the Orion Arm and its correlation to EM was also noted by Haverkorn et al. (2004), who compare EM and RM structure functions in the southern sky and see evidence for two spatially distinct Faraday screens, one of which they attribute to the Local Arm. The region was also investigated by Brown et al. (2007), who again find evidence for distinct Galactic arms. The field reversal region between the Orion and the Sagittarius arm in the northern sky is investigated by Ordog et al. (2017), among others. They discuss a diagonal progression of the reversal region from $(l, b)=\left(67^{\circ}, 4^{\circ}\right)$ to $(l, b)=\left(56^{\circ},-2^{\circ}\right)$, which is strongly visible in Fig. 13a. Especially interesting to us are the results of Bracco et al. (2019), who were able to produce a map of the linear dust polarization fraction using 3D analytical models of the magnetic field under the assumption of a helical magnetic field component. Their map shows strong morphological similarities to our $\delta$ field, with very little polarization in the regions of the Local Arm. As dust polarization probes the planar component of the magnetic field, this indicates that most of the magnetic field strength lies in the LOS component of the magnetic field. This can be confirmed by a comparison to the Planck dust polarization fraction map calculated from the Planck Stokes parameter maps in Fig. 14, which show similar features in the respective regions of the Local Arm.

The comparison of the amplitude field in Fig. 10c without the $\delta$ contributions with the $\epsilon$ field alone (see Fig. 10a) reveal the linear rescaling of the free-free contribution to the $\gamma$ and $\psi$ fields according to Eq. (19). This mostly results in a much less pronounced disk, which seems to be disfavored by the Faraday data.

Finally we discuss the sign field $\chi$ in Fig. 8a. Little has changed morphologically in this component compared to Fig. 8b. The absolute value of the field has decreased by approximately a factor of 4 . This factor was mostly absorbed into the new amplitude field, as discussed before. Interestingly enough, we can still discern the region of underdense Faraday data in the

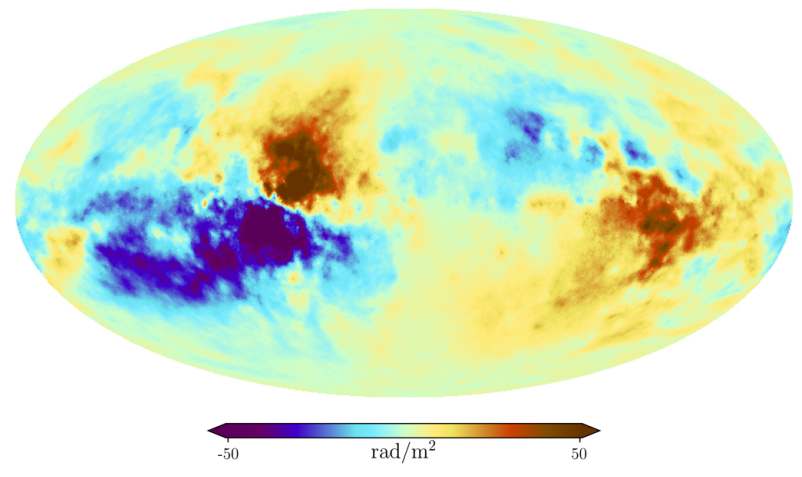

(a)

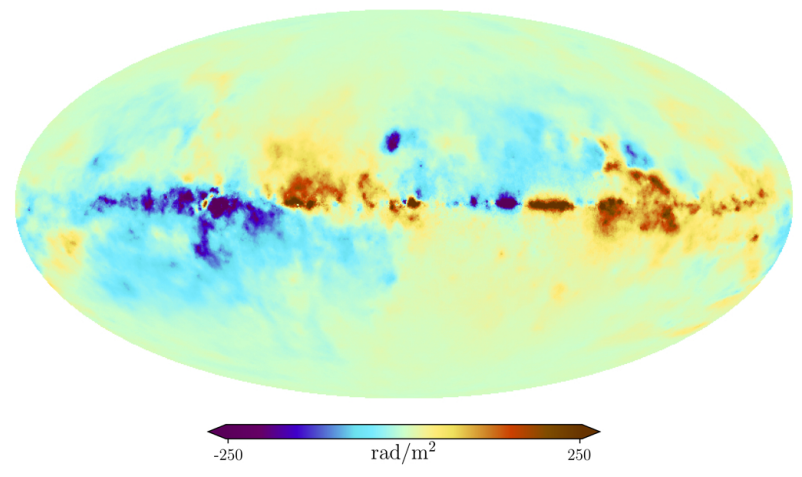

(b)

Fig. 13. Panel $a$ : Faraday sky containing only the amplitude contribution stemming from the field $\delta$ as defined in Eq. (21). This map is a rough estimate for the enhancement of the Faraday signal by the Orion arm. Panel $b$ : Faraday sky resulting from the complementary amplitude contributions (see also Fig. 10b).

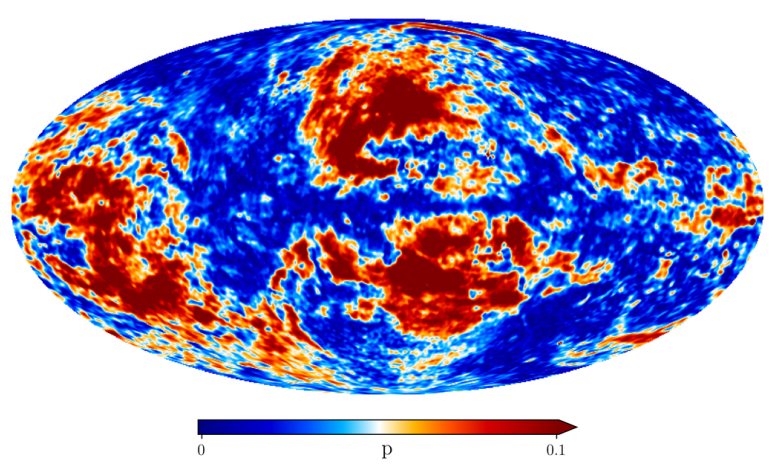

Fig. 14. Linear polarization fraction $p=\frac{\sqrt{Q^{2}+U^{2}}}{I}$ of dust calculated from the Planck polarization data. 


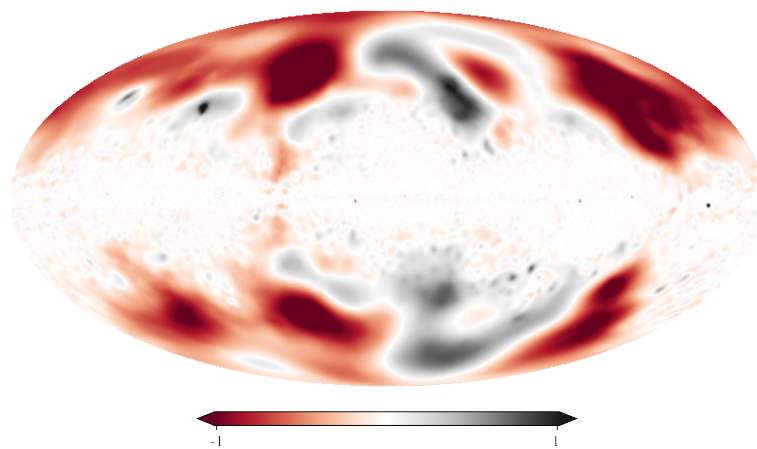

(a)

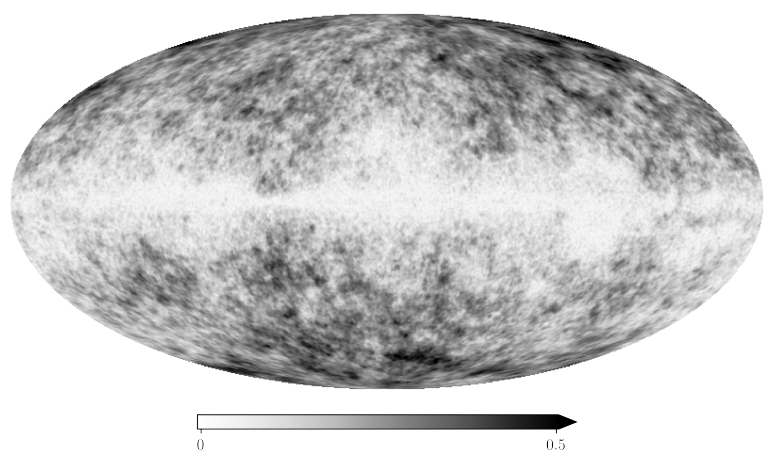

(b)

Fig. 15. Panel $a$ : difference between field $\epsilon$, as defined in Eq. (18) and inferred only by free-free data, and the same field resulting from the joint inference with Faraday data (reconstruction I). Panel $b$ : uncertainty of the $\epsilon$ field in reconstruction I. This demonstrates that the $\epsilon$ field is predominately determined by the free-free data irrespective of the simultaneous inference of Faraday sky. There are considerable deviations between the reconstructions near the Galactic poles. Panel $b$ shows that the reconstruction is also rather uncertain in these regions.

southern sky in this plot, which has very little structure in it. This is further evidence that the decrease in uncertainty in Fig. 5a in this region is caused by the now strongly determined amplitude field, while the small-scale structure of the sign of the Faraday depth is still only vaguely known. Apart from that, the field has again fully served its intended purpose, namely capturing the sign of the Faraday sky.

We now discuss the results of the inference of the free-free EM sky.

\subsubsection{The revised free-free map}

The revised free-free EM sky constrained by the Planck and Faraday data is shown in Fig. 9c, its logarithm is shown in Fig. 10a. We also ran a $\mathrm{EM}_{\mathrm{ff}}$ inference solely using the Planck data. The difference to Fig. 10a is shown in Fig. 15a. There is little difference between the maps, indicating that the systematic differences of the data sets were mostly absorbed by the second amplitude field $\delta$ and the rescaling fields $\gamma$ and $\epsilon$. Notable differences appear only towards the Galactic poles, where Planck has measured little to no free-free component, while considerable Faraday rotation exists. These regions are also subject to strong uncertainty in the reconstructed free-free EM, as indicated in Fig. 15b. The visual comparison of Fig. 9c to the data shown in Fig. 9a demonstrates good agreement. The

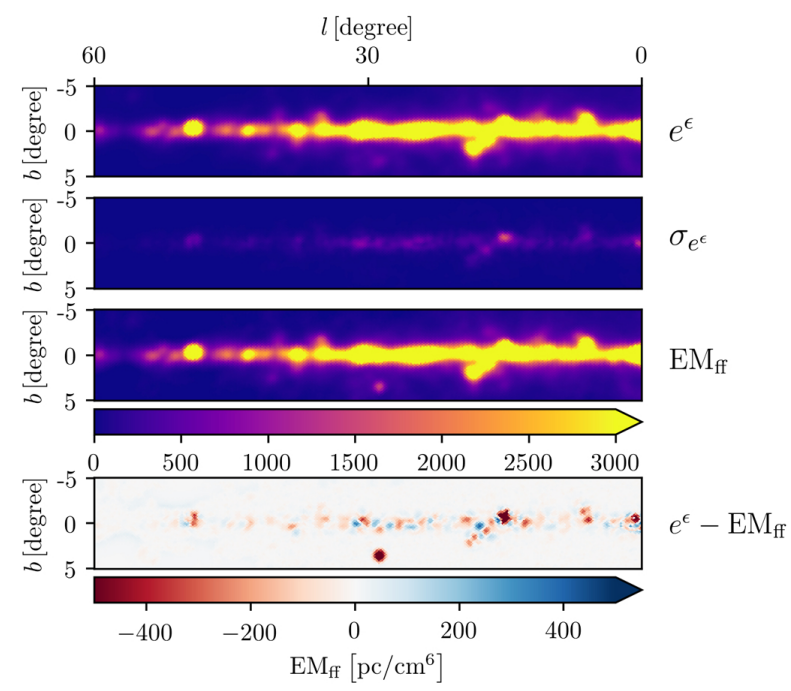

Fig. 16. Excerpt of the free-free reconstruction in the first slice, its uncertainty, followed by the same excerpt in the Planck free-free EM data. The residual is shown in the last row.

main difference is the lack of small structures far away from the disk, which were mostly absorbed into the noise.

Closer inspection reveals some small-scale deviations in the disk, which we want to investigate by showing an excerpt of the disk ranging from $0^{\circ}$ to $60^{\circ}$ longitude in Fig. 16. This shows approximately the same region as the first plot of Fig. 7 in Planck Collaboration XXV (2016), where the Planck team compares their free-free temperature map with independent measurements via the radio recombination line survey (RRL). While our reconstruction reproduced the overall morphology, some peaks in the picture experience deviations of around $20 \%$. In general our results seem to underestimate the amplitudes compared to Planck. It is noteworthy that the comparison between Planck and RRL resulted in a similar statement, as the Planck features were generally much more pronounced. We refrain from drawing physical conclusions from these discrepancies as they can only be resolved by a detailed discussion of the Planck data analysis, which is beyond the scope of this work. We note again that the Faraday data has little influence on this result. The changes are mainly driven by the noise estimation and the full consideration of the correlation structure of the free-free EM sky. We think this makes the inclusion of the correlation structure in the inference is an important step for any component separation algorithm, as also shown in Knollmüller \& Enßlin (2017).

\section{Summary and conclusion}

We infer the Galactic Faraday sky map using the same data as NO12, but using an updated algorithm. This involves the separation of the Faraday sky into the point wise product of a sign and amplitude field, which are supposed to mainly capture the sign and the Galactic profile of the Faraday sky, respectively. In the first part of the paper, we can largely confirm the previous results in map and power spectrum. Smaller differences can be explained by the algorithmic advancements. The biggest improvement here is the updated uncertainty map, where we were able to significantly reduce the error bars over the whole sky, especially in some locations in the Galactic disk.

In the further proceedings we found that the amplitude field has notable similarities to the Galactic free-free EM map. In the second part of the paper we therefore incorporated free-free EM 
data as a potential proxy for the Faraday amplitude field into our inference. This lead to a new Faraday map, which now is notably different to previous results. The Galactic disk is much more pronounced and uncertainties are largely reduced. Since a pronounced Galactic disk is reasonable and data constraints are now better satisfied than in any of the other approaches, we regard this map as the most reliable and therefore to be the main result of this work. Nonetheless, it should be noted that our error maps cannot incorporate the modeling uncertainties of our inference model. In addition, the component fields introduced in our model to resolve discrepancies between the two data sets reveal indicators for more LOS aligned magnetic field structures in the direction of the Orion arm. We also produce a denoised free-free EM map, which agrees with the Planck data, on which it is based to large extent, but which is free of many of the point source-like structures seen in the Planck data above the Galactic disk.

Of course any additional data will improve our work. Specifically, for the southern sky new RM data is already available and the analysis with our framework is under way. Looking farther into the future, the ongoing ASKAP-POSSUM survey (Gaensler et al. 2010) and the anticipated SKA polarization surveys will allow us to resolve the Faraday sky to an unprecedented sharpness of detail.

Acknowledgement. We would like to thank Brandon Hensley for pointing out that the field alignment effect is also visible in the Planck polarization data. Furthermore, we would like to thank the referee for the very constructive feedback, which greatly improved this paper.

\section{References}

Bennett, C. L., Larson, D., Weiland, J. L., et al. 2013, ApJS, 208, 20

Bonafede, A., Feretti, L., Murgia, M., et al. 2010, A\&A, 513, A30

Bonafede, A., Vazza, F., Brüggen, M., et al. 2013, MNRAS, 433, 3208

Boudjada, M. Y., \& Lecacheux, A. 1991, A\&A, 247, 235

Bracco, A., Candelaresi, S., Del Sordo, F., \& Brandenburg, A. 2019, A\&A, 621, A97

Braun, R., Oosterloo, T. A., Morganti, R., Klein, U., \& Beck, R. 2007, A\&A, 461,455

Brentjens, M. A., \& de Bruyn, A. G. 2005, A\&A, 441, 1217

Broten, N. W., Macleod, J. M., \& Vallée, J. P. 1988, Ap\&SS, 141, 303

Brown, J. C., Taylor, A. R., \& Jackel, B. J. 2003, ApJS, 145, 213

Brown, J. C., Haverkorn, M., Gaensler, B. M., et al. 2007, ApJ, 663, 258

Burn, B. J. 1966, MNRAS, 133, 67

Clarke, T. E. 2004, J. Korean Astron. Soc., 37, 337

Clarke, T. E., Kronberg, P. P., \& Böhringer, H. 2001, ApJ, 547, L111

Clegg, A. W., Cordes, J. M., Simonetti, J. M., \& Kulkarni, S. R. 1992, ApJ, 386, 143

Condon, J. J., Cotton, W. D., Greisen, E. W., et al. 1998, AJ, 115, 1693

Crutcher, R. M., Wandelt, B., Heiles, C., Falgarone, E., \& Troland, T. H. 2010, AJ, 725, 466

de Gouveia Dal Pino, E. M. 2006, AIP Conf. Ser., 875, 289

Dennison, B. 1979, AJ, 84, 725

Dineen, P., \& Coles, P. 2005, MNRAS, 362, 403

Dreher, J. W., Carilli, C. L., \& Perley, R. A. 1987, ApJ, 316, 611

Enßlin, T. A. 2019, Ann. Phys., 531, 1800127

Enßlin, T. A., \& Frommert, M. 2011, Phys. Rev. D, 83, 105014

Enßlin, T. A., \& Weig, C. 2010, Phys. Rev. E, 82, 051112

Enßlin, T. A., Frommert, M., \& Kitaura, F. S. 2009, Phys. Rev. D, 80, 105005

Eriksen, H. K., Jewell, J. B., Dickinson, C., et al. 2008, ApJ, 676, 10

Feain, I. J., Cornwell, T. J., Ekers, R. D., et al. 2011, ApJ, 740, 17

Feain, I. J., Ekers, R. D., Murphy, T., et al. 2009, ApJ, 707, 114

Frick, P., Stepanov, R., Shukurov, A., \& Sokoloff, D. 2001, MNRAS, 325, 649

Gaensler, B. M., Dickey, J. M., McClure-Griffiths, N. M., et al. 2001, ApJ, 549, 959

Gaensler, B. M., Haverkorn, M., Staveley-Smith, L., et al. 2005, Science, 307, 1610

Gaensler, B. M., Landecker, T. L., Taylor, A. R., \& POSSUM Collaboration 2010, in BAAS, Vol. 42, Amer. Astron. Soc. Meet. Abstr., \#215, 515

Gießübel, R., Heald, G., Beck, R., \& Arshakian, T. G. 2013, A\&A, 559, A27
Gregorini, L., Vigotti, M., Mack, K. H., Zoennchen, J., \& Klein, U. 1998, A\&AS, 133,129

Greiner, M., Schnitzeler, D. H. F. M., \& Enßlin, T. A. 2016, A\&A, 590, A59

Harvey-Smith, L., Madsen, G. J., \& Gaensler, B. M. 2011, ApJ, 736, 83

Haslam, C. G. T., Salter, C. J., Stoffel, H., \& Wilson, W. E. 1982, A\&AS, 47, 1

Haverkorn, M., Gaensler, B. M., McClure-Griffiths, N. M., Dickey, J. M., \& Green, A. J. 2004, ApJ, 609, 776

Haverkorn, M., Gaensler, B. M., McClure-Griffiths, N. M., Dickey, J. M., \& Green, A. J. 2006, ApJS, 167, 230

Heald, G., Braun, R., \& Edmonds, R. 2009, A\&A, 503, 409

Hennessy, G. S., Owen, F. N., \& Eilek, J. A. 1989, ApJ, 347, 144

Johnston-Hollitt, M. 2003, Detection of magnetic fields and diffuse radio emission in Abell 3667 and other rich southern clusters of galaxies/Melanie Johnston-Hollitt

Johnston-Hollitt, M., \& Ekers, R. D. 2004, unpublished [arXiv:astro-ph/0411045]

Johnston-Hollitt, M., Hollitt, C. P., \& Ekers, R. D. 2004, in The Magnetized Interstellar Medium, eds. B. Uyaniker, W. Reich, \& R. Wielebinski (Göttingen, Germany: Copernicus Publications), 13

Kato, T., Tabara, H., Inoue, M., \& Aizu, K. 1987, Nature, 329, 223

Kim, K.-T., Tribble, P. C., \& Kronberg, P. P. 1991, ApJ, 379, 80

Klein, U., Mack, K. H., Gregorini, L., \& Vigotti, M. 2003, A\&A, 406, 579

Knollmüller, J., \& Enßlin, T. A. 2017, Phys. Rev. E, 96, 042114

Knollmüller, J., \& Enßlin, T. A. 2019, ArXiv e-prints [arXiv:1901.11033]

Knollmüller, J., Steininger, T., \& Enßlin, T. A. 2017, ArXiv e-prints [arXiv:1711.02955]

Kooi, J. E., Fischer, P. D., Buffo, J. J., \& Spangler, S. R. 2017, Sol. Phys., 292, 56

Lallement, R., Capitanio, L., Ruiz-Dern, L., et al. 2018, A\&A, 616, A132

Lawler, J. M., \& Dennison, B. 1982, ApJ, 252, 81

Leike, R. H., \& Enßlin, T. A. 2019, A\&A, 631, A32

Ma, Y. K., Mao, S. A., Stil, J., et al. 2019, MNRAS, 1401, 3454

Mao, S. A., Gaensler, B. M., Stanimirović, S., et al. 2008, ApJ, 688, 1029

Mao, S. A., Gaensler, B. M., Haverkorn, M., et al. 2010, ApJ, 714, 1170

Mao, S. A., McClure-Griffiths, N. M., Gaensler, B. M., et al. 2012, ApJ, 755, 21

McClure-Griffiths, N. M., Dickey, J. M., Gaensler, B. M., et al. 2005, ApJS, 158, 178

Mestel, L. 1966, MNRAS, 133, 265

Minter, A. H., \& Spangler, S. R. 1996, ApJ, 458, 194

Oppermann, N., Junklewitz, H., Robbers, G., et al. 2012, A\&A, 542, A93

Oppermann, N., Junklewitz, H., Greiner, M., et al. 2015, A\&A, 575, A118

Ordog, A., Brown, J. C., Kothes, R., \& Landecker, T. L. 2017, A\&A, 603, A15

Oren, A. L., \& Wolfe, A. M. 1995, ApJ, 445, 624

Passot, T., \& Vázquez-Semadeni, E. 2003, A\&A, 398, 845

Planck Collaboration X. 2016, A\&A, 594, A10

Planck Collaboration XXV. 2016, A\&A, 594, A25

Pumpe, D., Gabler, M., Steininger, T., \& Enßlin, T. A. 2018, A\&A, 610, A61

Purcell, C. R., Gaensler, B. M., Sun, X. H., et al. 2015, ApJ, 804, 22

Remazeilles, M., Dickinson, C., Banday, A. J., Bigot-Sazy, M. A., \& Ghosh, T. 2015, MNRAS, 451, 4311

Roy, S., Rao, A. P., \& Subrahmanyan, R. 2005, MNRAS, 360, 1305

Rudnick, L., \& Jones, T. W. 1983, AJ, 88, 518

Selig, M., Bell, M. R., Junklewitz, H., et al. 2013, A\&A, 554, A26

Selig, M., Vacca, V., Oppermann, N., \& Enßlin, T. A. 2015, A\&A, 581, A126

Simard-Normandin, M., Kronberg, P. P., \& Button, S. 1981, ApJS, 45, 97

Smoot, G. F. 1998, ArXiv e-prints [arXiv:astro-ph/9801121]

Steininger, T., Dixit, J., Frank, P., et al. 2017, ArXiv e-prints [arXiv:1708.01073], unpublished

Stil, J. M., Taylor, A. R., \& Sunstrum, C. 2011, ApJ, 726, 4

Tabara, H., \& Inoue, M. 1980, A\&AS, 39, 379

Taylor, A. R., Gibson, S. J., Peracaula, M., et al. 2003, AJ, 125, 3145

Taylor, A. R., Stil, J. M., \& Sunstrum, C. 2009, ApJ, 702, 1230

Vallée, J. P. 2018, Ap\&SS, 363, 243

Van Eck, C. L., Brown, J. C., Stil, J. M., et al. 2011, ApJ, 728, 97

Vázquez-Semadeni, E. 2015, Astrophys. Space Sci. Lib., 407, 401

Vázquez, R. A., May, J., Carraro, G., et al. 2008, ApJ, 672, 930

Vernstrom, T., Gaensler, B. M., Rudnick, L., \& Andernach, H. 2019, ApJ, 878, 92

Vigotti, M., Gregorini, L., Klein, U., \& Mack, K. H. 1999, A\&AS, 139, 359

Wiener, N. 1966, Extrapolation, Interpolation, and Smoothing of Stationary Time Series (Cambridge, Mass.: Technology Press of the Massachusetts Institute of Technology)

Wrobel, J. M. 1993, AJ, 106, 444

Xu, J., \& Han, J.-L. 2014, Res. Astron. Astrophys., 14, 942

Xu, Y., Kronberg, P. P., Habib, S., \& Dufton, Q. W. 2006, ApJ, 637, 19

Xu, Y., Reid, M. J., Menten, K. M., et al. 2009, ApJ, 693, 413 


\section{Appendix A: Generic IFT concepts}

These appendices provide the details on the inference scheme used in this work. We start by introducing some core concepts of IFT. After that, the details of the correlation structure inference are explained. In this part we also show the equations for the full posterior of our model above. We close with some details on the posterior evaluation scheme used. Extended and more mathematically rigorous discussions on these topics can be found in Enßlin et al. (2009); Knollmüller et al. (2017); Enßlin (2019), and Knollmüller \& Enßlin (2019).

A generic expression for the posterior probability distribution for an inference problem can be written using Bayes theorem:

$\mathcal{P}(s \mid d)=\frac{\mathcal{P}(d \mid s) \mathcal{P}(s)}{\mathcal{P}(d)}$.

In our case the signal $s$ contains all fields that describe the abovementioned properties of the desired sky maps. The left-hand sight of Eq. (A.1) is commonly called the posterior. The righthand side consists of the prior $\mathcal{P}(s)$, the likelihood $\mathcal{P}(d \mid s)$ and the evidence $\mathcal{P}(d)$. The last is important for model comparison tests, but negligible in an inference algorithm that aims to find the optimal field realizations of $s$ for a given model as it does not depend on $s$, but only serves as a normalization constant.

We consider the information Hamiltonian, or negative logprobabilities, of the above equation,

$\mathcal{H}(s \mid d)=-\ln \mathcal{P}(s \mid d)$.

This is mostly for reasons of convenience, as the resulting sums of log-probabilities are usually numerically better behaved as the product of probabilities. Furthermore, there are nice analogies to statistical mechanics and field theory (hence the use of "Hamiltonian"), which result in a rich variety of techniques IFT can make use of.

Using the expressions for the likelihoods from Eqs. (7) and (17), the respective Gaussian priors similar to Eq. (9), and the terms stemming from the noise estimation models in Eq. (11), we can write down the posterior Hamiltonian assuming independent measurements of $\boldsymbol{d}=\left(d_{\phi}, d_{\mathrm{ff}}\right)^{T}$ :

$$
\begin{aligned}
\mathcal{H}(\boldsymbol{s} \mid \boldsymbol{d}) & \equiv-\log (\mathcal{P}(\boldsymbol{s} \mid \boldsymbol{d})) \\
& \hat{=} \sum_{d \in \boldsymbol{d}} \mathcal{H}(d \mid \mathbf{s})+\sum_{f \in \mathbf{s}} \mathcal{H}(f) \\
& \hat{=} \sum_{d \in \boldsymbol{d}} \frac{1}{2}\left(d-\mathcal{R}_{d} \boldsymbol{s}\right) \widetilde{N}_{d}^{-1}\left(d-\mathcal{R}_{d} \boldsymbol{s}\right)^{\dagger} \\
& +\frac{\beta_{d}}{\eta_{d}}+\left(\alpha_{d}+1\right) \ln \left(\eta_{d}\right)+\sum_{f \in \boldsymbol{s}} f^{\dagger} S_{f}^{-1} f .
\end{aligned}
$$

The vector $s \equiv(\ldots)^{T}$ contains all Gaussian random fields introduced in the previous sections, which are assumed to be a priori independent from each other. The symbol indicates the omission of unnecessary additive constants. This equation already contains most of the model. A crucial part is missing; specifically, we have not specified the prior covariances $S_{f}$. The modeling and inference of these are explained in the next section.

\section{Appendix B: Correlation structure model}

Again we do not follow the details in NO12, but use an updated algorithm for the correlation structure inference. A short discussion on the differences of the two approaches and potential implications on the spectrum follows at the end of this section. We verified our results via a comparison to the previous map only using the data used in NO12 in Sect. 2.5.

The assumption of statistical homogeneity and isotropy together with Gaussianity for a field $f$ implies via the WienerKhintchin theorem that the respective covariance is diagonal in harmonic space. We make use of this theorem as it allows us to describe the correlation structure via a field with at most $N_{\text {pix }}$ dimensions, as opposed to the $N_{\text {pix }}^{2}$ dimensions in the nonharmonic space. The correlation structure of a Gaussian field $f$ is then fully described via its power spectrum defined as

$C_{\ell}^{(f)}=\mathbb{P}_{\ell}\left\langle\widehat{f f^{*}}\right\rangle_{\mathcal{P}_{(x)}}=\mathbb{P}_{\ell} \frac{1}{2 \ell+1} \sum_{m}\left|\widehat{f_{\ell m}}\right|^{2}$,

where $\mathbb{P}_{\ell}$ is a projection operator onto spectral bands $\ell$ of the power spectrum. This operator simply gives us the possibility to reduce the degrees of freedom of the spectrum, it may very well be set to a unit matrix if, for example, rather sharp features are expected, such as characteristic frequencies.

For the inference, we follow the reparametrization method laid out in Knollmüller et al. (2017). We decompose the sky fields into

$f=\mathbb{Y} A_{f} \xi_{f}$,

where $\mathbb{Y}$ is the spherical harmonic transform. The amplitude fields $A_{f}$ contain the information on the correlation structure on the respective field. The fields $\xi_{f}$ are a priori white Gaussian fields with zero mean and unit covariance, and contain the information of the specific field configuration of the field $f$ given the correlation structure in $A_{f}$. Both fields live in harmonic space. We can show that the amplitude fields are related to the covariance via

$S_{f}=\mathbb{Y} A_{f} A_{f}^{\dagger} Y^{\dagger}$.

This formulation has immediate consequences for the Hamiltonian, as the prior terms are now independent of the correlation structure as

$f^{\dagger} S_{f}^{-1} f=\xi_{f}^{\dagger} \xi_{f}$,

using the above equations. For a more general discussion of the advantages of this approach we refer the reader to Knollmüller et al. (2017). Now we are left with the inference of the amplitude fields. They are modeled via a log-normal field $\tau_{f}$ to ensure positivity:

$A_{f}=\mathbb{P}_{\ell} e^{\frac{1}{2} \tau_{f}}$.

The inference then follows the approach of Arras et al. (in prep.), in which $\tau_{f}$ is again transformed according to the reparametrization method above into a white field $\xi_{f}$ and hyperparameters $\sigma_{f}$ and $l_{f}$, which steer the expected variability in the spectrum. The resulting hierarchical tree model is shown in Fig. B.1.

In NO12 the power spectrum was modeled via a log-normal field together with the product of an inverse gamma and a regularizing smoothness likelihood, while the flattening of the hierarchical structure demonstrated above was not performed.

We show a comparison in Sect. 2.5 to demonstrate that the new algorithm reproduces the inference of the correlation structure in NO12, and we also comment on the minor differences occurring between the two spectra. The full Hamiltonian for the 


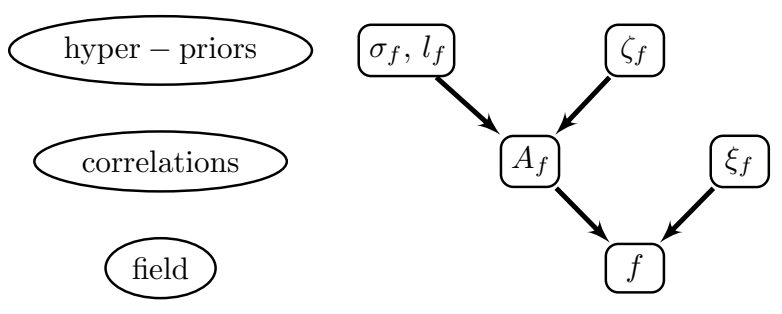

Fig. B.1. Hierarchical tree model for a generic Gaussian field $f$ on the sky. It is decomposed into an amplitude field $A_{f}$ containing the correlation structure and an a priori white Gaussian field $\xi_{f}$ connecting this correlation information to the actual spatial representation. $A_{f}$ on its own again depends on hyper-priors $\sigma_{f} l_{f}$ and another white field $\zeta_{f}$. This tree wraps up Appendix B and is the core building block of our correlation structure inference.

simultaneous inference of Faraday sky, the free-free sky, the correlation structure of their respective component fields, and noise correction terms is then as follows:

$$
\begin{aligned}
\mathcal{H}(\boldsymbol{s} \mid \boldsymbol{d}) & \hat{=} \sum_{d \in \boldsymbol{d}} \frac{1}{2}\left(d-\mathcal{R}_{d} \boldsymbol{s}\right) \widetilde{N}_{d}^{-1}\left(d-\mathcal{R}_{d} \boldsymbol{s}\right)^{\dagger} \\
& +\frac{\beta_{d}}{\eta_{d}}+\left(\alpha_{d}+1\right) \ln \left(\eta_{d}\right)+\sum_{f \in \boldsymbol{s}} \xi_{f}^{\dagger} \xi_{f}+\mathcal{H}\left(\tau_{f} \mid \zeta_{f}, \sigma_{f}, l_{f}\right) .
\end{aligned}
$$

The last term contains the hyper-priors for the $\tau_{f}$ inference and is described in detail in Arras et al. (in prep.).

\section{Appendix C: Evaluating the posterior}

The Hamiltonian in Eq. (B.6) fully describes our information on the problem. Unfortunately, the non-linearities in this expression imply that this posterior distribution is non-Gaussian, making the evaluation a non-trivial task, as a general analytic solution is not known to exist. Therefore, we need to choose an appropriate numerical evaluation scheme. To this end, variational Bayes approaches have proven to combine satisfying accurateness in their uncertainty estimates with speed and stability during the minimization (Knollmüller et al. 2017). Specifically, this implies that we minimize the Kullback-Leibler Divergence (KL)

$$
\begin{aligned}
\mathcal{D}_{\mathcal{K} \mathcal{L}}(\widetilde{\mathcal{P}}(s \mid d) \| \mathcal{P}(s \mid d)) & =\int \mathcal{D} s \widetilde{\mathcal{P}}(s \mid d) \ln \left(\frac{\widetilde{\mathcal{P}}(s \mid d)}{\mathcal{P}(s \mid d)}\right) \\
& =\langle\mathcal{H}(s \mid d)\rangle_{\widetilde{\mathcal{P}}(s \mid d)}-\langle\widetilde{\mathcal{H}}(s \mid d)\rangle_{\widetilde{\mathcal{P}}(s \mid d)},
\end{aligned}
$$

where $\widetilde{\mathcal{P}}$ is a Gaussian probability distribution and $\widetilde{\mathcal{H}}$ is the respective Hamiltonian. $\langle\ldots\rangle_{\widetilde{\mathcal{P}}}$ indicates an averaging procedure of the quantity in the brackets with respect to $\widetilde{\mathcal{P}}$. Minimizing the above expression can be interpreted as a fit of $\widetilde{\mathcal{P}}$ to $\mathcal{P}$. This approximated posterior is then easily evaluated via sampling due to its Gaussian nature.

Both the Hamiltonian and the KL functional can be conveniently implemented into NIFTy (Selig et al. 2013; Steininger et al. 2017; The NIFTy5 team et al. 2019, priv. comm.), which in its newest version then takes care of calculating the correct gradients via auto-differentiation and provides a library of suitable minimization schemes for optimizing Eq. (C.1). Convergence is assessed via predefined convergence criteria and the stability is tested via starting the inference from different (both deliberately and randomly chosen) initial conditions. For further details on the MGVI technique used here, see Knollmüller \& Enßlin (2019). 